\title{
The Variability and Rotation of Pre-main Sequence Stars in IC 348: Does Intracluster Environment Influence Stellar Rotation?
}

\author{
Stella Nordhagen ${ }^{1}$ \\ Department of Physics, Middlebury College, Middlebury, VT 05753 \\ snordhag@middlebury.edu \\ and \\ William Herbst, Katherine L. Rhode and Eric C. Williams \\ Astronomy Department, Wesleyan University, Middletown, CT 06459
}

\begin{abstract}
A variability study of the young cluster IC 348 at Van Vleck Observatory has been extended to a total of seven years. Twelve new periodic stars have been found in the last two years, bringing the total discovered by this program to 40. In addition, we confirm 16 of the periods reported by others and resolve some discrepancies. The total number of known rotation periods in the cluster, from all studies has now reached 70 . This is sufficient to demonstrate that the parent population of K5-M2 stars is rotationally indistinguishable from that in the Orion Nebula Cluster even though their radii are $20 \%$ smaller and they would be expected to spin about twice as fast if angular momentum were conserved. The median radius and, therefore, inferred age of the IC 348 stars actually closely matches that of NGC 2264, but the stars spin significantly more slowly. This suggests that another factor besides mass and age plays a role in establishing the rotation properties within a cluster and we suggest that it is environment. If disk locking were to persist for longer times in less harsh environments, because the disks themselves persist for longer times, it could explain the generally slower rotation rates observed for stars in this cluster, whose earliest type star is of class B5. We have also obtained radial velocities, the first for PMS stars in IC348, and $v$ $\sin i$ measurements for 30 cluster stars to assist in the study of rotation and as an independent check on stellar radii. Several unusual variable stars are discussed; in some or all cases their behavior may be linked to occultations by circumstellar
\end{abstract}

\footnotetext{
${ }^{1}$ REU summer student at Wesleyan University
} 
material. A strong correlation exists between the range of photometric variability and the slope of the spectral energy distribution in the infrared. Nineteen of the 21 stars with I ranges exceeding 0.4 mag show infrared evidence for circumstellar disks.

Subject headings: open clusters and associations: individual (IC 348) — stars: rotation — stars: pre-main-sequence

\section{Introduction}

IC 348 is a compact, young cluster in the Perseus clouds at a distance of about 300 pc (Cernis 1993). It contains $\sim 200$ members, with spectral types ranging from B5 to late $\mathrm{M}$ and about two dozen probable brown dwarfs that are also likely to be cluster members (Luhman et al. 2003, 2005b). It is especially important to star formation studies because it is relatively close to us, compact on the sky, well studied by a variety of methods, and has a nearly complete census of members. It is also intermediate in nature between the denser, more massive clusters, such as the Orion Nebula Cluster (ONC), and the looser associations, such as the Taurus $\mathrm{T}$ association. For a recent summary of work on the cluster, see the article by Herbst (2006) in The Handbook of Low Mass Star Forming Regions.

Photometric monitoring of young clusters has led to the determination of accurate rotation periods for about 2,000 pre-main sequence stars (see, for example, the review by Herbst et al. (2006)). The best-studied cases are the ONC and NGC 2264. Herbst \& Mundt (2005) have recently reviewed the data and shown that the evolution of rotation for stars in the 0.4-1.2 solar mass range may be understood in terms of two phenomena. About half of the stars of Orion age (the half which are already the more rapid rotators) spin up with essentially no loss of angular momentum all the way to the ZAMS. The other half continue to be slowed by interaction with circumstellar disks for times of up to 5 Myr and end up as very slow rotators on the ZAMS. This picture is based on only five clusters, two containing pre-main sequence stars and three containing ZAMS stars. Obviously it will be important to test it by obtaining data for more clusters and, in particular, clusters with different properties (such as stellar density and environment). IC 348 is a particularly useful cluster in this regard for the reasons discussed in the first paragraph.

The cluster has been photometrically monitored at Van Vleck Observatory (VVO) on the campus of Wesleyan University for seven years. Two previous papers in this series have reported on the progress of this program after one and five years, respectively (Herbst, Maley \& Williams 2000; Cohen, Herbst \& Williams 2004). Littlefair et al. (2005) have recently 
published results from a short (17 day) but intensive photometric monitoring program done with a larger telescope and going deeper than our work, and Kiziloğlu et al. (2005) have even more recently published the results of a six month monitoring program designed to search for variability in stars with X-ray counterparts. In this paper, we present data from an additional two years of photometric monitoring at VVO as well as a complementary study of radial velocities and $v \sin i$ measurements for 30 cluster members. We combine the results of all of the studies to discuss the rotation period distribution in the cluster and its significance for our understanding of angular momentum evolution in solar-like stars. A very recent infrared study of the cluster with Spitzer by Lada et al. (2006) allows us to correlate rotation and variability properties with the presence or absence of evidence for a disk. In addition, we discuss some unusual variable stars.

\section{Observations and Reductions}

\subsection{Photometry}

Observations of IC 348 at Wesleyan have been obtained between 1998 December 10 and 2005 March 17. Earlier papers in this series (Herbst, Maley \& Williams 2000; Cohen, Herbst \& Williams 2004) have reported results for the first five years; here we concentrate on the last two years. All data were obtained using a 1024 x 1024 Photometrics CCD camera attached to the $0.6 \mathrm{~m}$ Perkin telescope at Wesleyan University's Van Vleck Observatory. As each pixel covers $0.6^{\prime \prime}$, the field of view is $10.2^{\prime}$ on a side. On each clear night during the observing season, five consecutive one-minute exposures were taken through a Cousins $I$ filter, along with bias frames, dark frames, and twilight flats. When possible, this sequence of five object frames was repeated more than once per night. Preliminary reductions were conducted using standard Image Reduction and Analysis Facility (IRAF) tasks, and each set of five images was combined and shifted to the same position within less than one pixel to create an image with an increased dynamic range and an effective five-minute exposure time.

Differential aperture photometry was performed on a sample of 151 stars, listed in Cohen, Herbst \& Williams (2004), using the IRAF APPHOT package with an aperture radius of 7 pixels $\left(4.2^{\prime \prime}\right)$. The median level of sky background was determined using an annulus with inner and outer radii of 10 and 15 pixels, respectively. Due to the close proximity of other stars, a few stars in the sample may have contaminated photometry. A list of these objects can be found in Herbst, Maley \& Williams (2000).

As the observations were taken over a seven-year interval, it was beneficial to have a 
set of stable comparison stars that could be used reliably over the entire time span. For this reason, the six comparison stars established by Cohen, Herbst \& Williams (2004) were used. All six stars $(3,7,8,10,13$, and 17) display standard deviations of less than 0.01 mag in each season. This stability is likely due to the first five stars' proximity to the main sequence and the sixth star's non-cluster-member status. Instrumental differential magnitudes $(i)$ were computed for each star on each night relative to the average magnitude of comparison stars. These values were transformed to standard magnitude $(I)$ using the adopted transformation

$$
I=i+11.142+0.099(R-I)
$$

The values of $R-I$ used in the transformation are taken from Herbig (1998) when available and from Trullols \& Jordi (1997) otherwise; when no $R-I$ value was available from either source, an assumed $R-I$ value of 2.00 was used, following Cohen, Herbst \& Williams (2004).

Fig. 1 shows the range of each star as a function of its mean magnitude. As expected, there is a lower envelope that increases in range with decreasing brightness due to random error. At the bright end, our accuracy is about $0.005 \mathrm{mag}$, allowing us to detect real variations as small as $\sim 0.02 \mathrm{mag}$ for irregular variables, or even smaller for periodic variables; where the signal is concentrated into a narrow frequency band. For the fainter stars in our sample, the errors grow sufficiently large that it is not possible for us to detect periods, let alone real variability, even when present. We return to a discussion of other features of Fig. 1 in the Results section.

\section{2. $v \sin i$ Measurements}

\subsubsection{Observations}

The $v \sin i$ observations were obtained in 2004 February using the Hydra multiobject spectrograph on the 3.5 m WIYN telescope ${ }^{1}$ at Kitt Peak National Observatory near Tucson, Arizona. The Hydra instrument allows simultaneous spectroscopy of $\sim 100$ targets over a $1^{\circ}$ field. We used the same spectrograph setup as for a previous study of rotation in the ONC done by Rhode, Herbst, \& Mathieu (2001) [hereafter RHM]: the Bench camera, red fiber cable, and 316@63.4 echelle grating. This setup yielded a resolution of $\mathrm{R} \sim 21,500$. The spectra cover $\sim 6240-6540 \AA$ with a central wavelength of $6400 \AA$ and $0.144 \AA$ per pixel dispersion. The spectral resolution, using a typical slit profile full-width half-maximum of 2 pixels, is $13.5 \mathrm{~km} \mathrm{~s}^{-1}$ at $6400 \AA$.

\footnotetext{
${ }^{1}$ WIYN is a joint facility of the University of Wisconsin, Yale University, and the National Optical Astronomy Observatories.
} 
The $v \sin i$ sample was chosen to include a mix of both Classical T Tauri stars (CTTS) and Weak T Tauri stars (WTTS). We also wanted to observe roughly equal numbers of periodic stars and a "control" group of IC 348 members without measured periods. (As we note in what follows, several of the stars in the control group have subsequently had their rotation periods measured.) The general aim was to compare the $v \sin i$ distributions of these various samples with each other, as was done in RHM. The final set of target stars also depended strongly on limitations on how the Hydra fibers could be positioned. Given all of these constraints, 37 target objects were observed in two different fiber configurations, with 22 objects in the first configuration and 15 in the second. Both configurations included 18 fibers positioned on the blank sky, to allow for sky-subtraction in the spectral reduction process.

Five 1800-second exposures were taken in each configuration. Five objects from the Gliese Catalog of Nearby Stars (Gliese \& Jahreiss 1991), with spectral types roughly spanning the range of the target stars, were observed for the RHM study with the same spectrograph setup, and were used again here. Spectra of these Gliese stars (which have Gliese sequence numbers 15A, 75, 114, 144, and 411) were used as narrow-lined templates in the crosscorrelation process; their coordinates, magnitudes and spectral types are given in RHM.

\subsubsection{Initial Reductions}

Preliminary data reductions were performed on both the template and target star spectra using standard IRAF tasks such as ZEROCOMBINE, CCDPROC, and FLATCOMBINE. The IRAF task DOHYDRA was then used on the object frames to extract the spectra and perform flat-fielding, fiber throughput correction, wavelength calibration, and sky subtraction. The wavelength calibration was accomplished using observations of a ThAr comparison lamp taken before and/or after each set of target star integrations. Sky fibers with unusually high signal due to object contamination were eliminated from the sky subtraction process. The remaining sky spectra were then averaged to create a combined sky spectrum, using a sigma-clipping algorithm to eliminate cosmic rays.

After the DOHYDRA reductions were completed, the five individual integrations of each target star were scaled and combined into a single frame using the task SCOMBINE. This produced a single high signal-to-noise observation that was free of cosmic rays. The combined frames were then clipped so that the spectra contained only the region between

6275 and $6525 \AA$, to eliminate the lower signal-to-noise regions at the ends of some of the images. Finally, the individual object spectra were continuum-normalized before the crosscorrelation process. 


\subsubsection{Measuring Radial and Rotational Velocities}

Increasing rotational velocity causes the absorption lines in a stellar spectrum to become broader and shallower. When a broadened target star spectrum is cross-correlated against a narrow-lined spectrum that otherwise contains similar spectral features, the width of the Fourier cross-correlation peak depends upon the amount of broadening present in the target spectrum. The width of this peak can therefore be used to derive the star's projected rotational velocity. The position of the cross-correlation peak also provides a measure of the radial velocity of the star with respect to the template object, which can then be used to derive the target star's heliocentric radial velocity. We used the IRAF task FXCOR to perform cross-correlation of target star spectra against spectra from narrow-lined template stars and derive radial and rotational velocities.

The first step in deriving $v \sin i$ is to establish the relationship between the measured width of the cross-correlation peak and $v \sin i$; details of how this was done are given in RHM. Briefly, the narrow-lined template spectra of the Gliese stars were artificially "spun-up" to higher rotational velocities by convolving them with a theoretical rotation profile (Gray 1992). The broadened spectra were then cross-correlated with the original versions. The FWHM values of the resultant peaks were measured and the relationship between FWHM and $v \sin i$ was quantified for each template star.

To estimate $v \sin i$ of the target stars, each star's spectrum was cross-correlated against the spectrum of the template star having the closest spectral type. ${ }^{2}$ The FWHM of the cross-correlation peak was measured and translated into a $v \sin i$ value using the previouslyderived calibration curve for the template star. Heliocentric radial velocities for the target stars were determined during the cross-correlation step using the measured relative shift in the cross-correlation peak, the radial velocity of the template star from Gliese \& Jahreiss (1991), and the position, date, and time of the observation. The cross-correlation function can also show structure that may indicate that a star is a double-lined spectroscopic binary. In this case the presence of two sets of spectral lines can cause the normally Gaussian-shaped function peak to appear double-peaked, like two Gaussians superimposed on one another. During the analysis, the cross-correlation peaks were checked for evidence of possible binary structure.

The limit to which $v$ sin $i$ can be measured depends in large part on the size of the slit (fiber) image. The slit image width of our instrumental setup, determined by measuring

\footnotetext{
${ }^{2}$ The IC 348 spectral types used in this study come from Herbig (1998) when available or Luhman et al. (2003) otherwise.
} 
the FWHM of emission-line profiles in the comparison lamp spectra, typically ranged from 1.7 to 1.9 pixels in the middle of the spectrum to $\geq 2$ pixels on the ends of the spectrum. A 1.7-pixel spectral resolution corresponds to a $v \sin i$ of $11 \mathrm{~km} \mathrm{~s}^{-1}$, which we take as the approximate lower limit on our $v \sin i$ measurements. $v \sin i$ values in this paper that are slightly above this limit should be treated with caution, as they might be upper limits rather than actual velocity measurements.

\section{Results}

\subsection{Photometric Rotation Periods}

\subsubsection{Determination of Periods and Classification}

Periodic variations in the light curves of the sample stars were searched for by calculating a periodogram for each star using the method of Scargle (1982) and the formulation of Horne \& Baliunas (1986). This method can yield multiple possible periods for a single star, some of which are beat periods caused by the interference of the natural period and the one-day sampling interval and others of which are false alarms caused by non-periodic variations. In order to separate true periods from aliases and false positives, the following criteria were employed. First, a star's power-spectrum (as generated by the periodogram function) must display a peak with a normalized power greater than 7.0 and a false-alarm probability (FAP), as defined by Horne \& Baliunas (1986), of $2 \%$ or less. Second, the star must display one or more of the following characteristics: (1) a well-defined light curve showing clear periodic variation with small scatter, and/or (2) the highest periodogram peak (exceeding a power of 7.0) recurring in multiple seasons. Stars with periods between 0.9 and 1.1 days were eliminated from consideration, as they could merely be an artifact of the one-day sampling interval. With our sampling intervals we are simply not reliably sensitive to that frequency range. In cases when a star was determined to be periodic in the majority of seasons, a search for the same period at a slightly lower than 7.0 power was conducted and, when found, these periods were counted as real detections in those seasons as long as they agreed to within $2 \%$ with a period had been identified in at least two other seasons.

Applying these criteria, 40 of the 151 sample stars have now been determined to be periodic based solely on the VVO data set. Twenty-eight of these stars were already identified by Cohen, Herbst \& Williams (2004) and 12 are new detections reported here. They are listed in Table 1 and their phased light curves in one season are shown in Fig. 2. Three of these had already been reported periodic by Littlefair et al. (2005) and two by Kiziloğlu et al. (2005), although in one case the period reported by Kiziloğlu et al. (2005) is a beat 
period, not the fundamental period (see notes to Table 1).

Figure 1 shows the relationship between range in magnitude and average magnitude for all the stars in our sample. CTTS (squares) are defined here as K or M type stars with $W_{H \alpha}>11 \AA$, while WTTS (circles) are defined as $\mathrm{K}$ or M type stars with $W_{H \alpha}<11 \AA$. Spectral types and $\mathrm{H} \alpha$ equivalent widths are taken from Herbig (1998) or Luhman et al. (2003). It is evident that most of the stars found periodic by us (solid symbols) tend to have fairly small ranges and be confined to the magnitude region $I \sim 12-16$. It is also apparent that only one of the CTTS is periodic. The brightest cluster stars are of B to F spectral type and close to the main sequence; as such we do not expect to find periodic variations for them and do not. Periods are found for several of the relatively bright G-type stars. The fact that we do no find periods among the stars fainter than $\mathrm{I}=16$ is undoubtedly an artifact of the limitations imposed by the quality of our photometry: Littlefair et al. (2005) were able to go deeper with better precision and discovered many periodic stars (asterisks on the figure) among stars near the faint end of our sample.

On the other hand, the lack of detection of periods among the brighter stars ( $\mathrm{I}=14-16)$ with larger ranges, which are preferentially CTTS (see Fig. 1), is arguably not an artifact of our observing strategy. Littlefair et al. (2005), for example, had a higher density of data points over a short period of time and they did not detect new periodic variations among these stars. They argue that they found CTTS periodic variables that we missed because of their higher sampling frequency. However, we note that their new periodic CTTS are mostly fainter stars whose periodicity we probably missed because of the increased random errors in our photometry at those limits, not because of our sampling frequency. There remains a sample of relatively bright CTTS that have not revealed their periods, regardless of sampling frequency.

The lack of detectable periods among highly-variable stars (with the exception of star 73, see below) could be due either to the periodic variations being overwhelmed beyond detection by large-amplitude irregular variations or to a physical difference in stellar-spot presence or configurations among highly-variable stars. The vast majority of periodic stars detected by us are WTTS. While sampling frequency limitations could play some role, as argued above, such limitations were not responsible for our lack of detection of periodicity in the brighter CTTS. Littlefair et al. (2005) found periods for a number (15) of fainter CTTS, comprising about $30 \%$ of their periodic detections; these stars were too faint for us to find periods. Perhaps fainter CTTS reveal periodicity more readily than brighter ones. Another possibility is that the classification of CTTS based on $\mathrm{H} \alpha$ equivalent widths is somewhat more suspect for faint stars, as the lower average signal can exaggerate the prominence of their spectral lines. The average range of CTTS in our sample is about 0.46 mag, compared 
to $0.24 \mathrm{mag}$ for WTTS.

\subsubsection{Analysis of Periodic Behavior Over Time}

Assuming that T Tauri stars, like our own sun, demonstrate latitude drift of spots and differential rotation, one might expect to find changes in period and light-curve shape as stellar spots change in size and shape and move to different stellar latitudes. However, we were unable to find clear evidence of differential rotation in IC 348. In general, our sample shows remarkable consistency with regard to periodicity. Overall, the variation in the length of the period from season to season was very small - in all but two cases, the periods differed by less than $3 \%$ over the entire 7 -year time-span, with the majority differing by less than $1.5 \%$. This change is below what we consider to be significant, and is likely caused by slight changes in spot configurations or phase changes. Of the remaining two stars, one is a star which has only been found to be periodic in two seasons, and thus could be the result of aliasing or coincidental error, while the other varies by only $5.7 \%$ over 7 seasons of monitoring, a result that is only marginally significant. The consistency of period length in IC 348 stars seems to suggest that either the rotation rate of $\mathrm{T}$ Tauri stars does not vary with latitude, consistent with the rigid-rotator models of Kuker \& Rudiger (1997), or that T Tauri stellar-spots evolve or remain stable only at a certain limited range of stellar latitudes. However, that we do observe large variations in light-curve shape between different seasons seems to indicate a changing configuration of spots over a range of latitudes.

Despite constancy of period length over the monitoring time-span, all of the periodic stars do show slight changes in light-curve shape and amplitude from year to year. We have looked for examples of systematic cyclical changes, as one would expect from stellarspot cycles, and found none. Given that the duration of the spot cycle on the Sun is 11.1 years, continued monitoring will clearly be necessary to confirm or reject the possibility of similar stellar spot cycles on T Tauri stars. Two examples of phased light-curves over the 7 seasons are shown in Figures 3 and 4. The light-curves were phased according to the periods detected within each individual year, rather than using an average detected period, as we found the individual-year periods to be less scattered in almost all instances. Figure 3 shows an example of a star which was consistently periodic throughout the monitoring period, while Figure 4 gives an example of a period which disappeared and reappeared during the monitoring period.

The repeatability of periodic behavior among IC 348 studies is also confirmed by comparison between the periods detected through our monitoring program and others. Littlefair

et al. (2005) have reported data on a total of 50 rotation periods in IC 348. Eight of these 
are for stars too faint to be measured in our program, 11 were not detectable as periodic in our data (likely due to the better signal-to-noise ratio for the stars in the Littlefair et al. (2005) data: the mean magnitude of these undetectable periodic stars is $I \sim 15.4$, while that of our periodic stars is $\sim 13.8$ ), and 21 were determined to be periodic by both studies. For the remaining 10 stars, we can identify agreeing periods in our data, but we had not reported them as periodic because they fail our identification criteria, mostly by having too high a FAP. We can note these ten periods as confirmations of those found by Littlefair et al. (2005) - periods which we can identify as present in our data, but which do not show sufficiently prominent periodic variations for us to identify them independently. They are listed in Table 2. Littlefair et al. (2005) reported the same phenomenon for five of their stars, i.e. that we had reported them as periodic and that their data confirmed the periods but with a FAP that was too high to be regarded as definitive. Unfortunately, Littlefair et al. (2005) also took this to be evidence that our FAP criterion was more liberal than theirs. This is certainly not true. The reality is that both groups have used criteria that are conservative enough that real periods have avoided detection as a result of the selection restrictions. $\mathrm{T}$ Tauri stars vary in their amplitudes and light curve shapes from epoch to epoch resulting in natural variations in the power that one will obtain from the Lomb-Scargel periodogram. As a result, failure to detect a period in any given epoch at any given power level cannot be used to infer the accuracy of an FAP selection criterion.

Overall, the agreement with Littlefair et al. (2005) is excellent; of the 21 independently found periodic stars the two studies had in common (i.e. not counting the "confirmations"), 19 of the detected periods were in agreement to within the error of their period determinations, about 0.1 days. This is gratifying confirmation of the reliability of periodic variability studies. Of the remaining two stars, one (our number 14) is the result of the beat phenomenon, and we defer to the Littlefair et al. (2005) period of 1.63 days, since their tightly-spaced monitoring is likely better suited to distinguishing beat periods from true periods than our sparser sampling. For the other star (our number 12), Littlefair et al. (2005) reported a period of 10.8 days, while we detected a period of 2.24 days in 5 of our 7 monitoring years. Light curves of star 12 for all seven seasons are shown phased for the 2.2 day period in Fig. 5. There is no evidence of a 10.8 day period in our data for any of the sample years, while the 2.2 day period produces a well-defined light curve in 4 of 7 seasons. Additionally, our periodogram analysis shows no evidence for a longer period. Given that our data extend over seven years and include 274 nights of observing, while the Littlefair et al. (2005) study was conducted over a much narrower time range (17 nights of data taken over a span of 26 days), we conclude that $\sim 2.24$ days is the correct period for this star.

A second, even more recent study by Kiziloğlu et al. (2005) offers an additional opportunity for comparision-again, with excellent agreement. This five month study was able to 
confirm 17 of the periods identified in Cohen, Herbst \& Williams (2004) within 1\%; in addition, 18 new periods were detected. Eight of these stars were also studied in our monitoring program, and for three of them we have since found periods. One of these agrees with that found by Kiziloğlu et al. (2005), while two (HMW 18 and HMW 143) do not, but follow the relationship $1 / p_{1}=1-1 / p_{2}$, marking one study's period as a beat period. As our data extend over a much longer period of time and offer stronger support for the periods detected in this study than in the Kiziloğlu et al. (2005) study, we conclude that our measurement is the more accurate one and list these stars as new detections. Of the remaining 5 detections by Kiziloğlu et al. (2005), 3 can be confirmed in our data, though not with a low enough FAP to be treated as independent detections.

\subsubsection{Period Distributions}

Figure 6 shows the distribution of periods in our sample, as well as the effects of eliminating the earliest (pre-K5) and latest (post-M2) stars from the distribution. The main characteristics of the complete IC 348 sample distribution are an absence of periods shorter than one day, a small number of periods longer than 10 days, and a dip in the distribution of periods between 3 and 5 days. The first peak of the distribution (around 2 days) falls substantially when the distribution is limited to stars earlier than spectral class M2.5, corresponding to mass $>0.25-0.4 M_{\odot}$, and nearly disappears when the distribution is further limited to spectral types of $\mathrm{K} 5$ and later (mass $<\sim 1.2 \mathrm{M}_{\odot}$ ), demonstrating that nearly all of the earlier-type sample stars are fast rotators (period $\leq 3$ days). This supports the inverse relationship between rotation and mass for the more massive stars, found in Cohen, Herbst \& Williams (2004). It is also evident that most of the later-type (low mass) stars in the sample also have relatively short periods ( $<6$ days), similar to what is found in the ONC and NGC 2264 (Herbst \& Mundt 2005). One sees clearly that, in this cluster, it is the intermediate mass stars (around 0.5-1 solar mass) that spin the slowest.

The distribution of detected periods for stars with spectral types earlier than M2.5 in IC 348 is shown in the left hand panels of Fig. 7 in comparison with like distributions for the very young ( 1 Myr) ONC and slightly older (2-4 Myr) NGC 2264. The ONC data is from Herbst et al. (2002), classified based on the spectral types of Hillenbrand (1997), while the NGC 2264 data is from Lamm et al. (2004), classified based on the spectral types of Rebull, Wolff, \& Strom (2004). The IC 348 and ONC distributions show clearly similar doublepeaked structures with peaks around 2 and 8 days; a Kolmogorov-Smirnov (K-S) Test on these distributions indicates that they are likely ( $\sim 72 \%$ probability) drawn from the same population. The NGC 2264 distribution, on the other hand, is much more skewed towards 
fast rotators; the difference between the IC 348 and NGC 2264 distributions is significant at a $2 \sigma$ level, with a $3.28 \%$ probability of being drawn from the same parent population.

The distributions are compared for stars of spectral types K5-M2 in the right-hand panels of Fig. 7. Though there appear to be some differences between the IC 348 and ONC distributions, particularly a dearth of early rotators in IC 348, a K-S Test shows that this difference is insignificant ( $57 \%$ probability of sharing the same parent population), largely due to the small size of the IC 348 sample. The difference between the IC 348 and NGC 2264 distributions, however, is highly significant, with only a $0.4 \%$ chance of the two samples being derived from the same parent population. The significance of these results for our understanding of the rotational evolution of solar-like stars is discussed in Section 4.

\subsubsection{Atypical Periodic and Irregular Variables}

In addition to periodic variability, many types of non-periodic pre-main sequence variability have been identified over the years, including FU and EX Orionis stars (FUors and EXors), as well as type II, and type III (UXor) variables (Herbig 1977, 1989; Herbst et al. 1994; Herbst \& Shevchenko 1999). Sixteen irregular variables in IC 348, mostly of type II, were identified and discussed by Cohen, Herbst \& Williams (2004); all these stars were monitored for an additional two seasons in this study. Most of these stars continued their irregular behavior, with one particularly remarkable continuance observed for star 15 (to be documented in a forthcoming paper.) Cohen, Herbst \& Williams (2004) identified one star (number 47) as both periodic and irregular at different epochs. At that time, this K8 WTTS with I 14.7 had been seen as periodic in only one season (1998-1999). In the next four seasons, it displayed no clear periodogram peak, but in 2000-01 and 2001-02 it displayed a light-curve with several deeper minima, apparently fading by $\sim 1 \mathrm{mag}$ in 1 day, then

quickly recovering to full brightness, a type of behavior more characteristic of an UXor than a typical WTTS. The new data show no more irregularity, but clear periodic variations at a period within about 0.1 day of that detected in the first monitoring season. This star's odd behavior is shown in the top two panels of Fig. 8. It certainly warrants further study in order to determine whether its irregular behavior continues and deduce a possible cause. Perhaps it is linked to the similarly odd star CB 34V (Tackett, Herbst \& Williams 2005).

A second periodic star which also displayed irregular variation was found in this study: star 73, shown phase-plotted for two years in the bottom two panels of Fig. 8 and also noted as irregular in Littlefair et al. (2005). Star 73, an M2 WTTS with an IR excess of $\Delta(I-K)=0.64$ and a THICK disk classification based on the slope of its infrared SED (Lada et al. 2006), has displayed periods of duration $7.59 \pm 0.08$ days in four of the 7 
monitoring seasons, and a period of 7.61 days at a slightly higher FAP in the 7th season. These periodic variations, however, are on a much larger amplitude scale $(\sim 1.3 \mathrm{mag})$ than any other periodic star, as shown in Fig. 1, where star 73 is the clear periodic outlier. In order to explain a decrease in brightness on this scale (by about a factor of three) using the typical model of a rotating spotted surface, the star would have to have either a very hot stellar spot or a cool stellar spot taking up the vast majority of the surface area on one face. Additionally, the light curve of star 73 takes a different shape than any other periodic star, showing the sort of steep decline immediately followed by a steep return to the original magnitude typically associated with eclipsing binaries. This leads us to believe that while this star does vary in a periodic way, the cause of this variation may not be the rotation of a spotted surface, as initially assumed. A spectrum of this star was included in our $v \sin i$ study and showed no unusual structure; additional study, particularly spectroscopic study, of this star would help to shed light on the cause of its periodic and non-periodic variation. As the star has an optically thick circumstellar disk according to the slope of its infrared SED (Lada et al. 2006), it is reasonable to suppose that, unlike the WTTS, we are witnessing the effects of rotation of a rather stable accretion hot spot. It is somewhat reminiscent of the odd behavior exhibited by the CTTS AA Tau (Bouvier et al. 2003).

\section{2. $v \sin i$ Results}

Spectra of 37 stars were obtained for the purpose of measuring $v \sin i$ and 30 of these produced reliable results. For the remaining seven, the spectra lacked sufficient signal to produce a clear cross-correlation peak. Half of the 30 stars with reliable cross-correlations yielded $v \sin i$ values less than or equal to our estimated resolution limit of $11 \mathrm{~km} \mathrm{~s}^{-1}$, so the $v \sin i$ values for these stars are upper limits.

Errors on our $v \sin i$ values were calculated using the $r$ parameter of Tonry \& Davis (1979), which is a measure of the signal-to-noise ratio of the peak of the cross-correlation function. Tonry \& Davis showed that velocities measured with cross-correlation should have errors that are proportional to $(1+r)^{-1}$, and Hartmann et al. (1986) found in a study of Taurus-Auriga and Orion that $\pm v \sin i /(1+r)$ was a good approximation of the $90 \%$ confidence level of their $v \sin i$ measurements. Here, as in RHM, we have adopted $\pm v \sin i /(1+r)$ as an estimate of the $1 \sigma$ errors on our $v \sin i$ values.

To explore whether this is a reasonable assumption, as well as to investigate possible systematic errors, two of us (S.N. and K.R.) independently executed the cross-correlation procedure to derive $v \sin i$ for the program stars. When the FXCOR task is run, the user interactively fits a Gaussian function to the cross-correlation peak and decides exactly how 
the fit should be done (i.e., which data points to include in the fit). This has a direct impact on the FWHM of the best-fit Gaussian function and therefore on the measured $v \sin i$. For stars with $v \sin i$ above our $11 \mathrm{~km} \mathrm{~s}^{-1}$ resolution, the values measured by both of us agreed within the $\pm v \sin i /(1+r)$ error estimate in all cases. This suggests that the $\pm v \sin i /(1+$ $r$ ) value is large enough to account for this particular source of systematic measurement error. Additional possible sources of systematic error in our $v \sin i$ measurement process include slight mismatches between the spectral features of program stars and the crosscorrelation template stars, and a bias toward larger $v \sin i$ due to line blending in the stellar spectra. Both of these issues were explored in depth by RHM using WIYN/Hydra data taken with the identical spectrograph setup used here, and with program stars of similar effective temperatures to the IC 348 targets and the same set of template stars. Based on that analysis, we conclude that the $\pm v \sin i /(1+r)$ error estimate is large enough to account for possible systematic uncertainties in the current data set as well.

Two target stars (HMW 20 and HMW 45) showed structure in their cross-correlation peaks that might indicate that they are double-lined spectroscopic binaries (SB2s). In both cases, the cross-correlation peak had the expected Gaussian shape, but with a prominent "bump" or second, lower-amplitude Gaussian peak on the side. In addition to $v$ sin $i$, we also measured the target stars' radial velocities as part of the cross-correlation process. These radial velocity measurements are, to our knowledge, the first for the pre-main-sequence stars in IC 348. The distribution of these radial velocities is shown in Figure 9. There are three apparent outliers in the distribution: two of the stars (not plotted) have negative radial velocities and are the possible SB2s identified via structure in their cross-correlation functions; the other is HMW 23. The mean heliocentric radial velocity for our stellar sample is $15.9 \pm 0.8 \mathrm{~km} \mathrm{~s}^{-1}$ if we exclude the two SB2 candidates and $16.5 \pm 0.6 \mathrm{~km} \mathrm{~s}^{-1}$ if we also exclude the outlier HMW 23. These values are close to the Snow et al. (1994) heliocentric radial velocity estimate of approximately $12-15 \mathrm{~km} \mathrm{~s}^{-1}$ for the supernova shell in which IC 348 is embedded.

The $v \sin i$ measurements for the 30 stars with reliable cross-correlation results are given in Table 3. The table lists sequence numbers for the stars (HMW and Luhman numbers), heliocentric radial velocity, weighted mean $v \sin i$ (calculated using the independent $v \sin i$ measurements done by S.N. and K.R.) and the error on the mean $( \pm v \sin i /(1+r)$, where here $r$ is the mean Tonry \& Davis $r$ value from the two measurements). Stars with $v \sin i<$ $11 \mathrm{~km} \mathrm{~s}^{-1}$ have " $\leq 11.0$ " in the $v \sin i$ column. Seven stars that were observed but that did not yield $v \sin i$ measurements (i.e., their cross-correlation results were unreliable) are not listed in the table; they are HMW 31, 80, 88, 101, 110, 141, and 154. A histogram showing the distribution of $v \sin i$ for the 30 stars is shown in Figure 10. 


\subsubsection{Rotation Periods and $v \sin i$ Measurements}

Assuming that the periodic behavior observed in the sample stars is the result of the rotation of spotted surfaces, we would expect to see a strong negative correlation between period and $v$ sin $i$ 's, in that stars with shorter periods should display faster rotational velocities. This relationship is shown in Fig. 11; although the stars with intermediate-length periods seem to display slightly lower $v \sin i$ values than would be expected, the difference in $v$ $\sin i$ between the two extremes of the period distribution is striking and offers strong support for the spotted-surface assumption. (A similar conclusion was reached by both Stassun et al. (1999), based on an observed correlation between line width and angular velocity, and RHM, based on a highly significant period and $v \sin i$ correlation, for pre-main-sequence stars in Orion.)

In addition to a direct period $-v$ sin $i$ comparison, we can use the measured rotation periods, in combination with radius estimates, to derive equatorial rotation velocities from the equation $v_{e q}=2 \pi R / P$ and compare these values to the $v \sin i$ measurements. The radii were derived from the relationship $L=4 \pi r^{2} \sigma T^{4}$, with the bolometric luminosities and effective temperatures coming from Luhman et al. (2003). For the stars for which Luhman et al. (2003) did not report these values, the effective temperatures were estimated based on spectral type using the scale of Cohen \& Kuhi (1979), and the luminosities were calculated using the formulation of Hillenbrand (1997) with a distance modulus of 7.5 (Herbig 1998). The bolometric corrections for this calculation were taken from Bessel (1991) and Bessel \& Brett (1988), while the intrinsic stellar colors are those of Bessel \& Brett (1988). A comparison of $v \sin i$ with the estimated rotational velocity shows the expected relationship. Despite the relatively small number of data points, there is an obvious correlation between $v \sin i$ and $v_{e q}$, and nearly all the stars respect the $v \sin i=v_{e q}$ limit within the estimated error. This result again helps confirm our underlying assumptions about $\mathrm{T}$ Tauri star rotation and periodic behavior. There is a slightly anomalous concentration of stars above the $v \sin i=v_{e q}$ line around $v \sin i \sim 10$, but as these stars all have $v \sin i$ measurements near or below our resolution limit, we conclude that this is likely simply an artifact of the measurement process, and that our $v \sin i$ results conform to previous assumptions about pre-main-sequence rotation.

The radii used in determining $v_{e q}$ are listed in column 6 of Table 3. They should be treated cautiously, as they are highly sensitive to errors in luminosity and effective temperature. In addition, column 7 lists the minimum radii $(R \sin i)$ derived from the relationship $P v \sin i=2 \pi R \sin i$. Fig. 12 plots the relationship between the two quantities. Although there are fairly large deviations between the two for individual stars, likely due to the im-

precision of the luminosity and effective temperature measurements used in determining the 
radii, overall the data points scatter around the line of equality. The mean calculated radius for the sample stars is $R=1.75 R_{\odot}$, while the mean $R \sin i$ for stars with $v \sin i$ measurements above the limit of $11 \mathrm{~km} / \mathrm{s}$ is $1.62 R_{\odot}$. We note that the mean radius (and, therefore, age) of the K5-M2 stars in IC 348 is nearly identical to that found for NGC 2264 (1.7 $\left.\mathrm{R}_{\odot}\right)$ and significantly less than what is found for the ONC $\left(2.1 \mathrm{R}_{\odot}\right)$, as derived by Herbst \& Mundt (2005).

\section{The Links Between Rotation Period, Photometric Variability and Circumstellar Disks}

The Spitzer Infrared Telescope is revolutionizing our understanding of star formation in many ways. Not the least of these is its ability to separate stars with infrared excess emission indicative of active accretion disks from those without it. The longer lever arm provided by this telescope's ability to probe further into the infrared with good signal-tonoise than has been possible heretofore has proven crucial to this endeavor. An excellent example of the value of such data for rotation studies is the work of Rebull et al. (2006) who show clearly that in the ONC, rapidly rotating stars do not have disks, while more slowly rotating stars may or may not have them. Since the popular disk locking theory for slowing the rotation of stars (Königl 1991) predicts, in its simplest form, some sort of correlation between rotation rate and the presence of a disk, we have investigated this in the present sample using Spitzer data from Lada et al. (2006). They compute the slope of the spectral energy distribution (SED) in the infrared for cluster stars and use that to divide the stars into three classes, termed STAR, ANEMIC and THICK. The STAR class indicates no infrared excess and, therefore, no evidence for a circumstellar disk. ANEMIC and THICK refer to infrared SED slopes indicative of mild and substantial infrared excess, respectively. We have also investigated the possible link between large amplitude irregular variability and accretion disks. First, we discuss the periodic stars, then the irregular variables.

\subsection{Periodic Stars}

It has been known since the work of Edwards et al. (1993) that there is a possible correlation between near-infrared excess (usually calculated as an excess in the I-K color index) and rotation. While the result has been challenged and the correlation is relatively weak and scattered for known reasons, it is nonetheless established at a very high level of statistical significance in the ONC (Herbst et al. 2002). Unfortunately, because of the natural scatter and nature of the correlation it requires a lot of stars to establish this in any cluster 
and there are relatively few stars available in IC 348. Furthermore, one must restrict such investigations to a fairly narrow mass range since rotation period is not just a function of infrared properties, it is also a strong function of mass. Here we follow previous work in the ONC and NGC 2264, as summarized recently by Herbst \& Mundt (2005), and concentrate on the spectral class range of $\mathrm{G}$ to $\mathrm{M} 2$.

Figure 13 shows the slope of the infrared portion of the SED, calculated by Lada et al. (2006) plotted against the rotation frequency (inverse of the period). On this plot, the slow rotators are to the left and rapid rotators to the right. There is a small natural gap at a frequency of 0.3 , corresponding to a rotation period of about 3.3 days. This plot may be compared directly with the ONC as shown in the references cited above. While the number of stars is too small to be of statistical significance it is, nonetheless, notable that the general form of the distribution of points is quite similar to what is seen in the ONC. Namely, the rapid rotators tend to have less infrared excess (larger negative slopes), while the slower rotators have a mixture of stars with substantial excesses indicative of optically thick accretion disks and stars without such disks.

Because of the nature of this distribution and the small numbers involved it is not easy to quantify the statistical significance of any possible relationship between IR SED and rotation period. However, if we adopt the disk class assignments made by Lada et al. (2006), the ratios of THICK:ANEMIC:STAR, for the rapid rotators (i.e period less than 3.3 days) are 1:1:8, while among the slow rotators they are 8:2:11. A one-sided Fisher's Exact Test yields a probability of only $14 \%$ that rotation and IR SED are uncorrelated based on these numbers. Other statistical tests, including a Monte Carlo simulation, suggest the probability is only $7 \%$ or less, but these rely on the sample size being large enough and that may not be the case here. Obviously these data alone would not constitute significant independent evidence for a disk-rotation link, but they do add some support to the picture because they are consistent with what is found in more populous clusters. In IC 348, as in the ONC and NGC 2264, slow rotators among the more massive stars are more likely to show infrared excesses indicative of optically thick disks than rapid rotators. Note that the interpretation of slow rotators without infrared excesses is that they are stars that have recently lost their disks and not yet had time to spin up. This is plausible because the time for a disk to disappear is probably the viscous accretion time scale, which is of order $10^{5}$ years, while the spin-up time is an order of magnitude larger. We conclude that the infrared data are consistent with the view that disk-locking is operating and has operated in IC 348 to produce the large range in currently observed rotation rates, just as it has in the ONC (and NGC $2264)$. 


\subsection{Irregular Variables}

Fig. 14 displays the relationship between a star's infrared properties and its variability range (which, as shown in Fig. 1, is largest for the irregular variables.) Evidently, there is a strong and highly statistically significant correlation between our measured photometric range for a star and its SED slope in the infrared from Lada et al. (2006). The sense of the correlation is that stars with evidence for circumstellar disks tend to have larger photometric ranges, exactly what we expect based on the canonical picture of CTTS variability as arising from unsteady accretion luminosity. Of the 21 stars in our sample with I magnitude ranges exceeding $0.4 \mathrm{mag}, 19$ have SED slopes indicative of ANEMIC or THICK disks. Large amplitude photometric variability is an excellent predictor of the presence of a circumstellar disk.

On the other hand, the absence of large amplitude variability does not necessarily preclude the presence of a disk. Many low amplitude variables are classified as ANEMIC or THICK by Lada et al. (2006). Presumably these are stars that were, for one reason or another, accreting at a steadier rate or less actively accreting during the observing epoch. It will be interesting to learn on what time scale they change this behavior and why, but that will require additional monitoring. These findings confirm the basic expectation about variability among T Tauri stars that CTTS will vary by larger amounts and more irregularly due to unsteady accretion, presumably from a circumstellar disk, while WTTS variability is predominantly from the rotational modulation of a spotted photosphere.

\section{Discussion: A Role for Environment in Establishing Initial Rotation Rates of Stars?}

Finally, we consider the origin of the fact that the period distribution in IC 348 of the G-M2 stars is similar to that of the ONC but significantly different from NGC 2264. The first thought would be that IC 348 and the ONC share a similar age. However, this is probably not true based on our best estimate of the radii of the pre-main sequence stars. Concentrating on the narrow spectral range K5-M2, and using the luminosities and effective temperature given by Luhman et al. $(2003,2005 \mathrm{a})$ we find that the average $R$ of these stars is $1.67 \pm 0.17 R_{\odot}$. This may be compared to values of 2.1 and $1.7 \mathrm{R}_{\odot}$ for the $\mathrm{ONC}$ and $\mathrm{NGC}$ 2264, respectively (Herbst \& Mundt 2005). These estimates, of course, do depend on the assumed distances to the clusters involved, and the reader is referred to Herbst (2006) for a discussion of the distance to IC 348 and its error. Most of the debate has been over whether the cluster could be closer than the adopted $300 \mathrm{pc}$, which would make the stars smaller

and older. With the commonly adopted distances, IC 348 is very close in age, as judged by 
mean radius of pre-main sequence stars, to NGC 2264 and significantly older than the ONC. These radii differences correspond to an age factor of about 2: IC 348 and NGC 2264 are about twice as old as the ONC. For a representative ONC age of 1 Myr, that makes IC 348 about 2 Myr old.

One would have expected that, all things being equal, the rotation period distribution of IC 348 would match that of NGC 2264, not the ONC. Clearly, something besides mass and age is responsible for influencing the rotation of stars in different clusters. An obvious possibility is the lifetime of disks, since it is disks that evidently control rotation during the pre-main sequence stage. To understand the observations one would require that disks last for a longer time in IC 348 than they do in either of the other clusters mentioned. This seems quite reasonable since the other clusters have $\mathrm{O}$ stars within them, while the earliest type star in IC 348 is of spectral class B5. The lack of ionizing photons means that the environment of the pre-main sequence stars is less hostile to disks than in the more massive clusters. This, in turn may mean that the disks could survive longer in the smaller cluster.

Our suggestion would be, therefore, that the rotation of stars will be influenced by the cluster environment in which they form. In particular, if it is a dense cluster with O stars then the disks will not last long and the stars will spin up earlier and more of them will end up as generally faster rotators on the ZAMS. In sparse clusters or associations, where disk lifetimes may be longer, one would expect more disk braking and, therefore, a larger proportion of slower rotators when they reach the ZAMS. This suggestion is consistent with the observations (although not the precise interpretation) of B-type stars in young clusters and the field, as discussed recently by Strom, Wolff \& Dror (2005). Obviously it will require many more observations of many more clusters and associations to ascertain whether this proposal has any merit.

\section{Summary and Conclusions}

This study has confirmed several beliefs about $\mathrm{T}$ Tauri star variability and rotation based on other clusters, identified some interesting stars worthy of continued observation and provided a challenge to the simplest picture of rotational evolution of low mass stars. In particular, the new data confirm that WTTS variability arises primarily from the rotation of a spotted photosphere and that the large amplitude (greater than 0.4-0.5 mag in I) irregular variability of CTTS is linked to the presence of an accretion disk. This study also verifies the accuracy, reliability and remarkable consistency of periods of pre-main sequence stars over time scales of many years and confirms the link between rotation and photometric period through comparison with $v \sin i$ data. 
The most unusual variable in IC 348 is HMW 15 and it is the subject of a separate, forthcoming contribution. Also notable is HMW 73, the only star to show large amplitude periodic behavior. This may be the result of rotation of a stable accretion hot spot or more unusual variable behavior.

Finally, we have shown here that the rotation period distribution of stars in the 0.4-1.2 solar mass range in IC 348 matches that of the ONC but contains a higher proportion of slow rotators than is seen in NGC 2264. Since the age of IC 348 is closer to NGC 2264 than the ONC, this suggests that age and mass alone are not sufficient to predict the rotation properties of stars in an extremely young cluster. We suggest that environment may also be a factor, as Strom, Wolff \& Dror (2005) have also proposed based on their v sin i study of $\mathrm{h}$ and $\chi$ Persei. If disk-locking controls rotational evolution for stars in this mass and age range then the longer accretion disks can persist, the longer the stars can continue to resist spin-up due to contraction with conservation of angular momentum. Perhaps in the less harsh environment of IC 348, whose earliest type star is of B5 and not a prodigious source of ultraviolet photons, the disks can persist longer than in NGC 2264. If so, the stars may continue to reflect an ONC-like distribution for times scales of order $2 \mathrm{My}$.

Continued study of this, and similar clusters, will be needed to ascertain whether this environment argument has merit. Because the rotation period distribution is so broad (and mass dependent) for young clusters, one requires a large number of stars to determine it and to distinguish significant differences among clusters. Obviously, it will continue to be of interest to monitor the variations of peculiar young stars such as HMW 15 and HMW 73 since they undoubtedly have much to tell us about the processes of accretion and disk evolution in solar-like stars.

It is a pleasure to thank the Wesleyan students who carried out the observations. S. N.'s work was supported by the National Science Foundation under Grant No. AST-0353997 to Wesleyan University, supporting the Keck Northeast Astronomy Consortium. K. R. was supported by an NSF Astronomy \& Astrophysics Postdoctoral Fellowship award under AST0302095. This material is based upon work supported by the National Aeronautics and Space Administration under Grant NNG05GO47G issued through the Origins of Solar Systems Program to W. H.

\section{REFERENCES}

Bessel, M.S. 1991, AJ, 101, 662 
Bessel, M.S. \& Brett, J.M. 1988, PASP, 100, 1134

Bouvier, J., Grankin, K. N., Alencar, S. H. P., Dougados, C., Fernandez, M. et al. 2003, A\&A409, 169

Carpenter, J. M. 2002, AJ, 124, 1593

Cernis, K. 1993, Baltic Astronomy, 2, 214

Chandrasekhar, S., \& Münch, G. 1950, ApJ, 111, 142

Cohen, R. E., Herbst, W. \& Williams, E. C. 2004, AJ, 127, 1602

Cohen, M., \& Kuhi, L.V. 1979, ApJS, 41, 743

de Zeeuw, P. T., Hoogerwerf, R., de Bruijne, J. H. H., Brown, A. G. A. \& Blaauw, A. 1999, AJ, 117, 354

Edwards, S., Strom, S., Hartigan, P., Strom, K. M., Hillenbrand, L. A. et al. 1993, AJ, 106, 372

Eislöffel, J., Froebrich, D., Stanke, T. McCaughrean, M. J. 2003, ApJ, 595, 259

Gliese, W., \& Jahreiss, H. 1991, Third Catalogue of Nearby Stars (Heidelberg: Astron. Rechen-Inst.)

Gray, D.F. 1992, The Observation and Analysis of Stellar Photospheres (Cambridge: Cambridge Univ. Press)

Haisch, K. E., Lada, E. \& Lada, C. J. 2001, AJ, 121, 2065

Hartmann, L., Hewett, R., Stahler, S., \& Mathieu, R.D. 1986, ApJ, 309, 275

Herbig, G. H. 1977, ApJ, 217, 693

Herbig, G. H. 1989, in ESO Workshop on Low Mass Star Formation and Pre-Main Sequence Objects, ed. B. Reipurth (Garching: ESO), 233

Herbig, G. H. 1998, ApJ, 497, 736

Herbst, W. 2006, in The Handbook of Low Mass Star Forming Regions, edited by B. Reipurth, in press.

Herbst, W. Eisloffel, J., Mundt, R. \& Scholz, A. 2006, in Protostars and Planets V., edited by B. Reipurth, in press. 
Herbst, W., Herbst, D.K., Grossman, E.J., \& Weinstein, D. 1994, AJ,108,1906

Herbst, W., Bailer-Jones, C. A. L., \& Mundt, R. 2001, ApJ, 554, L197

Herbst, W., Bailer-Jones, C. A. L., Mundt, R., Meisenheimer, K., \& Wackermann, R. 2002, A\&A396, 513

Herbst, W., Maley, J. A. \& Williams, E. C. 2000, AJ, 120, 349

Herbst, W., \& Mundt, R., 2005, ApJ633, 967

Herbst, W., \& Shevchenko, V. S. 1999, AJ, 118, 1043

Hillenbrand, L. A. 1997 AJ, 113, 1733

Horne, J.H., \& Baliunas, S.L. 1986, ApJ, 302, 757

Kiziloğlu, Ü., Kiziloğlu, N., \& Baykal, A. 2005, AJ, 130, 2766

Kuker, M. \& Rudiger, G. 1997, A\&A, 328, 253

Königl, A. 1991, ApJ370, L39

Lada, C. J., Muench, A. A., Luhman, K. L., Allen, L., Hartmann, L. et al. 2006, AJ, 131, 1574

Lamm, M. H., Bailer-Jones, C. A. L., Mundt, R., Herbst, W. \& Scholz, A. 2004, A\&A, 417, 557

Littlefair, S.P, Naylor, T., Burningham, B., \& Jeffries, R.D. 2005, MNRAS, 358, 341

Luhman, K. L. 1999, ApJ, 525, 466

Luhman, K. L., Lada, C. J., Hartmann, L., Muench, A. A., Megeath, S. T., Allen, L. E., Myers, P. C., Muzerolle, J., Young, E. \& Fazio, G. G. 2005, ApJ, in press

Luhman, K. L., Lada, E., Muench, A. A., \& Elston, R. J. 2005, ApJ, 618, 810

Luhman, K. L., McLeod, K. K. \& Goldenson, N. 2005, ApJ, 623, 1141

Luhman, K. L., Stauffer, J. R., Muench, A. A., Rieke, G. H., Lada, E. A., Bouvier, J. \& Lada, C. J. 2003 ApJ, 593, 1093

Mainzer, A. K. \& McLean, I. S. 2003, ApJ, 597, 555

McCaughrean, M. J., Rayner, J. T. \& Zinnecker, H. 1994, ApJ, 436, L189 
Muench, A. A., Lada, E. A., Lada, C. J., Elston, R. J., Alves, J. F., Horrobin, M., Huard, T. H., Levine, J. L., Raines, S. N. \& Roman-Zuniga, C. 2003, AJ, 125, 2029

Preibisch, T., Stanke, T. \& Zinnecker, H. 2003, A\&A, 409, 147

Preibisch, T. \& Zinnecker, H. 2001, AJ, 122, 866

Preibisch, T. \& Zinnecker, H. 2002, AJ, 123, 1613

Preibisch, T. \& Zinnecker, H. 2004, A\&A, 422, 1001

Rebull, L. M., Wolff, S. C., \& Strom, S. E. 2004, AJ127, 228

Rebull, L. M., Stauffer, J. R., Megeath, T., Hora, J. \& Hartmann, L. 2006, BAAS37, 185.08

Rhode, K.L., Herbst, W. \& Mathieu R.D. 2001, AJ, 122, 3258

Scargle, J.D. 1982, ApJ, 263, 835

Snow, T.P., Hanson, M.M., Seab, G.C., \& Saken, J.M. 1994, ApJ, 420, 632

Stassun, K.G., Mathieu, R.D., Mazeh, T., \& Vrba, F.J. 1999, AJ, 117, 2941

Strom, S. E., Wolff, S. C. \& Dror, D. H. A. 2005, AJ, 129, 809

Tackett, S., Herbst, W. \& Williams, E. 2003, AJ, 126, 348

Tonry, J., \& Davis, M. 1979, AJ, 84, 1511

Trullols, E. \& Jordi, C. 1997, A\&A, 324, 549 
Table 1. Newly Detected Periodic Stars at VVO

\begin{tabular}{ccc}
\hline \hline Star Number & Period (Days) & Notes \\
\hline 9 & 2.54 & $a$ \\
17 & 12.98 & \\
18 & 1.84 & $b$ \\
21 & 8.98 & $c$ \\
33 & 5.09 & \\
36 & 4.44 & \\
46 & 2.08 & $c$ \\
64 & 6.69 & $c$ \\
91 & 6.89 & \\
94 & 2.29 & \\
111 & 8.04 & \\
143 & 4.50 & $d$
\end{tabular}

eat period reported by Kiziloğlu et al. (2005).

aSimilar period detected in Kiziloğlu et al. (2005).

${ }^{\mathrm{b}} \mathrm{B}$

${ }^{\mathrm{c}}$ Similar period detected in Littlefair et al. (2005).

${ }^{\mathrm{d}}$ Could be the result of periodic variation in either star 143 or 144, which are photometrically indistinguishable. 
Table 2. Stars Detected as Periodic Both at VVO and by Littlefair et al. (2005).

\begin{tabular}{|c|c|c|c|}
\hline Star Number & Period (Days) & Littlefair et al. Period (Days) & Notes \\
\hline 12 & 2.24 & 10.81 & $a$ \\
\hline 14 & 2.54 & 1.63 & $b$ \\
\hline 16 & 5.22 & 5.21 & \\
\hline 21 & 8.98 & 9.11 & \\
\hline 26 & 3.02 & 3.01 & \\
\hline 27 & 2.67 & 2.65 & \\
\hline 29 & 8.61 & 7.93 & * \\
\hline 30 & 7.57 & 7.55 & \\
\hline 31 & 3.09 & 3.08 & \\
\hline 32 & 4.94 & 4.90 & \\
\hline 34 & 6.35 & 8.43 & $*, c$ \\
\hline 37 & 13.37 & 14.00 & $*$ \\
\hline 39 & 9.67 & 10.16 & \\
\hline 41 & 7.00 & 7.12 & \\
\hline 45 & 2.41 & 2.39 & \\
\hline 46 & 2.08 & 2.09 & \\
\hline 50 & 5.48 & 5.36 & \\
\hline 51 & 11.51 & 12.43 & \\
\hline 52 & 10.77 & 11.22 & \\
\hline 60 & 7.09 & 6.96 & \\
\hline 64 & 6.87 & 6.84 & \\
\hline 69 & 3.26 & 3.27 & \\
\hline 74 & 2.27 & 2.25 & * \\
\hline 81 & 3.99 & 3.98 & \\
\hline 98 & 3.51 & 3.58 & * \\
\hline 107 & 2.14 & 1.90 & $*, d$ \\
\hline 122 & 1.54 & 1.54 & * \\
\hline 133 & 6.35 & 6.31 & * \\
\hline 134 & 8.69 & 8.56 & \\
\hline 135 & 7.47 & 7.48 & $*$ \\
\hline 142 & 2.98 & 1.68 & $*, d$ \\
\hline
\end{tabular}

*An asterisk in the 'Notes' column indicates that for this star, we were able to confirm the period detected by Littlefair et al (2005) - i.e., a similar period was present in our data, though it did not meet our criteria for independent detection.

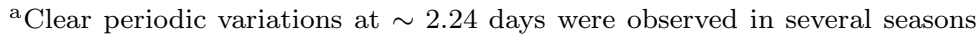
of data for this star, whereas we found no evidence of a 10.8 day period in any of the seven seasons. Given that our data span a much larger time range, and would thus be well suited to detecting any long-duration ( $\sim 10$ day) period that existed, we conclude that 2.24 days is the more accurate measurement.

${ }^{\mathrm{b}}$ In this case, we expect that our period may be a beat period, and defer to the shorter period identified by Littlefair et al (2005.)

${ }^{\mathrm{c}}$ Although there is a fairly large discrepancy between the two periods, the 6.5 day period shows up strongly in our data, albeit below our normal detection criteria. 
${ }^{\mathrm{d}}$ Here we have detected a beat period for the Littlefair period, indirectly confirming the period identified in that study. 
Table 3. $\quad v \sin i$ Results

\begin{tabular}{|c|c|c|c|c|c|c|c|}
\hline HMW \# & Luhman \# & $\begin{array}{c}v_{\text {helio }} \\
\left(\mathrm{km} \mathrm{s}^{-1}\right)\end{array}$ & $\begin{array}{c}v \sin i \\
\left(\mathrm{~km} \mathrm{~s}^{-1}\right)\end{array}$ & $\begin{array}{l}\text { Period } \\
\text { (days) }\end{array}$ & $\begin{array}{c}R \\
\left(R_{\odot}\right)\end{array}$ & $\begin{array}{c}R \sin i \\
\left(R_{\odot}\right)\end{array}$ & Notes \\
\hline 12 & L29 & $15.0 \pm 4.3$ & $47.1 \pm 3.3$ & 2.24 & 2.01 & 2.08 & \\
\hline 16 & L36 & $23.2 \pm 2.0$ & $14.5 \pm 0.9$ & 5.22 & 2.31 & 1.50 & \\
\hline 20 & L5 & $-4.9 \pm 4.0$ & $26.5 \pm 2.8$ & $\ldots$ & $\ldots$ & $\ldots$ & $a$ \\
\hline 22 & $\ldots$ & $17.5 \pm 3.8$ & $11.8 \pm 1.4$ & $\ldots$ & $\ldots$ & $\ldots$ & \\
\hline 23 & L37 & $0.2 \pm 2.5$ & $\leq 11.0$ & $\ldots$ & $\ldots$ & $\ldots$ & \\
\hline 26 & $\ldots$ & $17.1 \pm 2.4$ & $19 . \overline{1} \pm 1.4$ & 3.02 & $\ldots$ & 1.14 & \\
\hline 29 & L32 & $18.1 \pm 2.8$ & $\leq 11.0$ & 7.93 & 2.39 & 1.31 & \\
\hline 34 & $\ldots$ & $14.0 \pm 4.4$ & $\leq 11.0$ & 8.43 & $\ldots$ & 1.38 & \\
\hline 39 & $\ldots$ & $15.0 \pm 3.3$ & $\leq 11.0$ & 9.67 & 1.75 & 1.05 & \\
\hline 40 & L125 & $15.7 \pm 3.4$ & $11 . \overline{8} \pm 1.2$ & 8.36 & 1.51 & 1.95 & \\
\hline 41 & $\ldots$ & $19.2 \pm 1.9$ & $\leq 11.0$ & 7.00 & 1.70 & 1.05 & \\
\hline 42 & L86 & $13.3 \pm 5.3$ & $11 . \overline{1} \pm 2.0$ & 6.56 & 1.78 & 1.44 & \\
\hline 44 & L65 & $18.7 \pm 1.8$ & $\leq 11.0$ & 16.40 & 1.68 & 2.66 & \\
\hline 45 & $\mathrm{~L} 23$ & $-9.4 \pm 5.3$ & $30 . \overline{9} \pm 3.8$ & 2.41 & 3.01 & 1.47 & $a$ \\
\hline 48 & L113 & $18.1 \pm 4.5$ & $\leq 11.0$ & $\ldots$ & $\ldots$ & $\ldots$ & \\
\hline 50 & $\ldots$ & $17.0 \pm 3.1$ & $12 . \overline{2} \pm 1.2$ & 5.48 & $\ldots$ & 1.32 & \\
\hline 51 & $\ldots$ & $16.4 \pm 4.2$ & $\leq 11.0$ & 11.51 & 0.99 & 2.37 & \\
\hline 52 & $\ldots$ & $18.0 \pm 2.9$ & $\leq 11.0$ & 10.77 & 1.74 & 2.14 & \\
\hline 53 & $\ldots$ & $14.3 \pm 4.0$ & $\leq 11.0$ & 13.48 & $\ldots$ & 2.90 & \\
\hline 56 & L75 & $14.1 \pm 5.7$ & $13.9 \pm 2.3$ & $\ldots$ & $\ldots$ & $\ldots$ & \\
\hline 59 & L41 & $13.9 \pm 4.8$ & $\leq 11.0$ & $\ldots$ & $\ldots$ & $\ldots$ & \\
\hline 65 & L61 & $24.9 \pm 5.7$ & $17.3 \pm 2.7$ & $\ldots$ & $\ldots$ & $\ldots$ & \\
\hline 73 & $\ldots$ & $14.4 \pm 4.3$ & $\leq 11.0$ & 7.58 & 1.19 & 0.69 & $b$ \\
\hline 75 & L40 & $18.9 \pm 3.8$ & $13.7 \pm 1.5$ & $\ldots$ & $\ldots$ & $\ldots$ & \\
\hline 78 & L45 & $18.8 \pm 5.2$ & $42.9 \pm 3.6$ & $\ldots$ & $\ldots$ & $\ldots$ & \\
\hline 81 & L91 & $11.5 \pm 5.3$ & $11.5 \pm 1.8$ & 3.99 & 1.64 & 0.91 & \\
\hline 133 & L60 & $15.8 \pm 6.7$ & $14.7 \pm 2.8$ & 6.31 & 1.31 & 1.83 & $c$ \\
\hline 134 & $\ldots$ & $11.1 \pm 7.9$ & $\leq 11.0$ & 8.69 & 1.18 & 1.65 & \\
\hline 152 & L67 & $16.9 \pm 2.9$ & $\leq 11.0$ & $\ldots$ & $\ldots$ & $\ldots$ & \\
\hline 153 & L63 & $14.7 \pm 4.7$ & $\leq 11.0$ & $\ldots$ & $\ldots$ & $\ldots$ & \\
\hline
\end{tabular}

a Structure in the cross-correlation function indicates that this star is a possible double-lined spectroscopic binary.

${ }^{\mathrm{b}}$ There is some uncertainty about the periodicity of this star — see Section 3.1.4.

${ }^{\mathrm{c}}$ Period from Littlefair et al. (2005). 


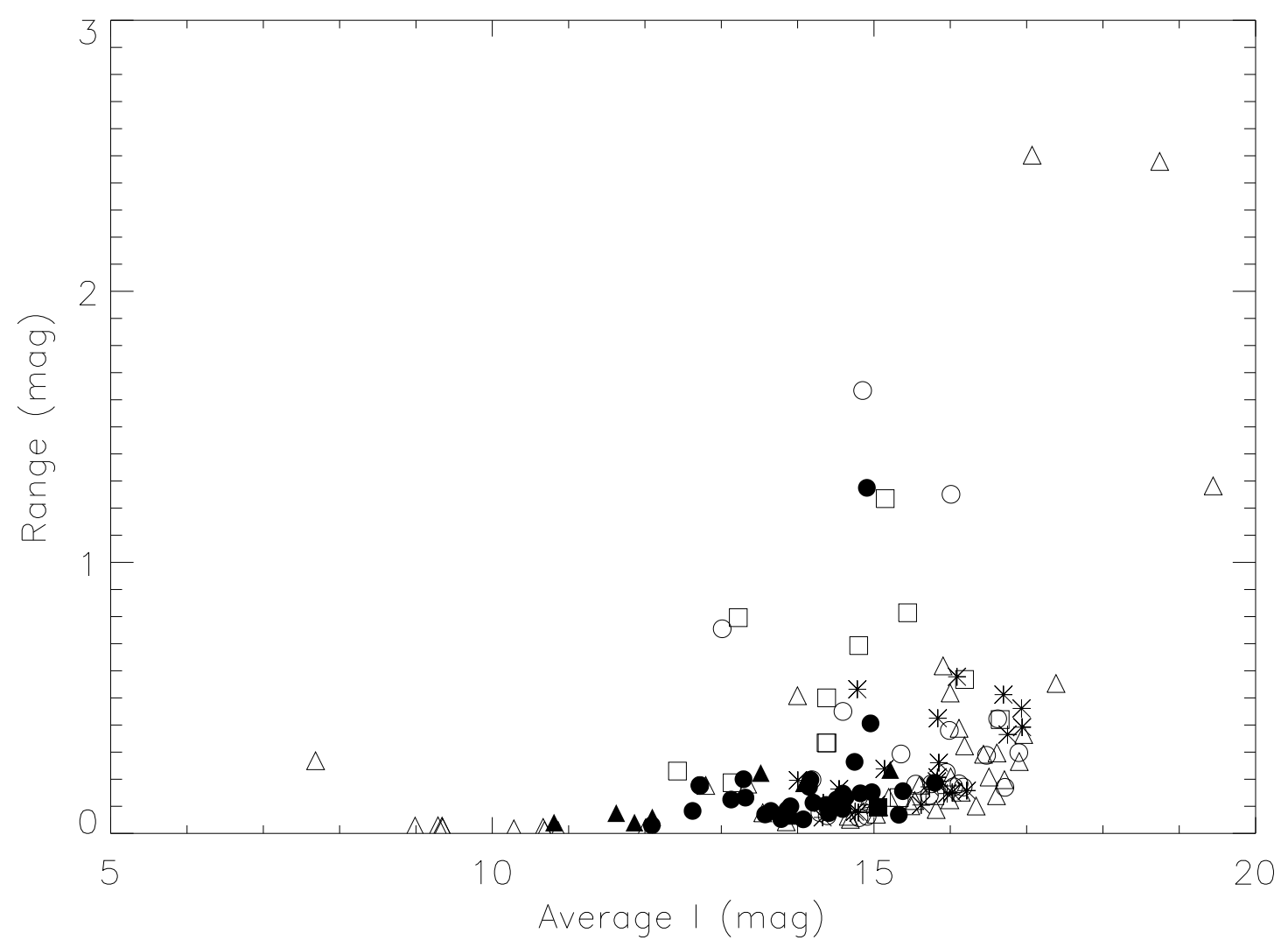

Fig. 1.- Range v. Magnitude for all the stars in our photometry sample, excepting those which are photometrically contaminated by nearby stars. WTTS are plotted as circles, CTTS are plotted as squares, and unclassified stars as triangles. Plotting symbols for the periodic stars are filled, while those of the non-periodic stars are empty. The absence of periods among the highly variable stars (excepting star 73) is evident, as is the lack of periods among both the brightest and the faintest stars. The sample stars determined to be periodic by Littlefair et al. (2005) only are plotted as asterisks. The majority of these newly-determined periodic stars are at the faint end of the magnitude distribution, supporting our belief that the lack of detected periods among stars in our sample with $I>16$ is due primarily to signal-to-noise limitations of our photometry and not to the monitoring frequency. 

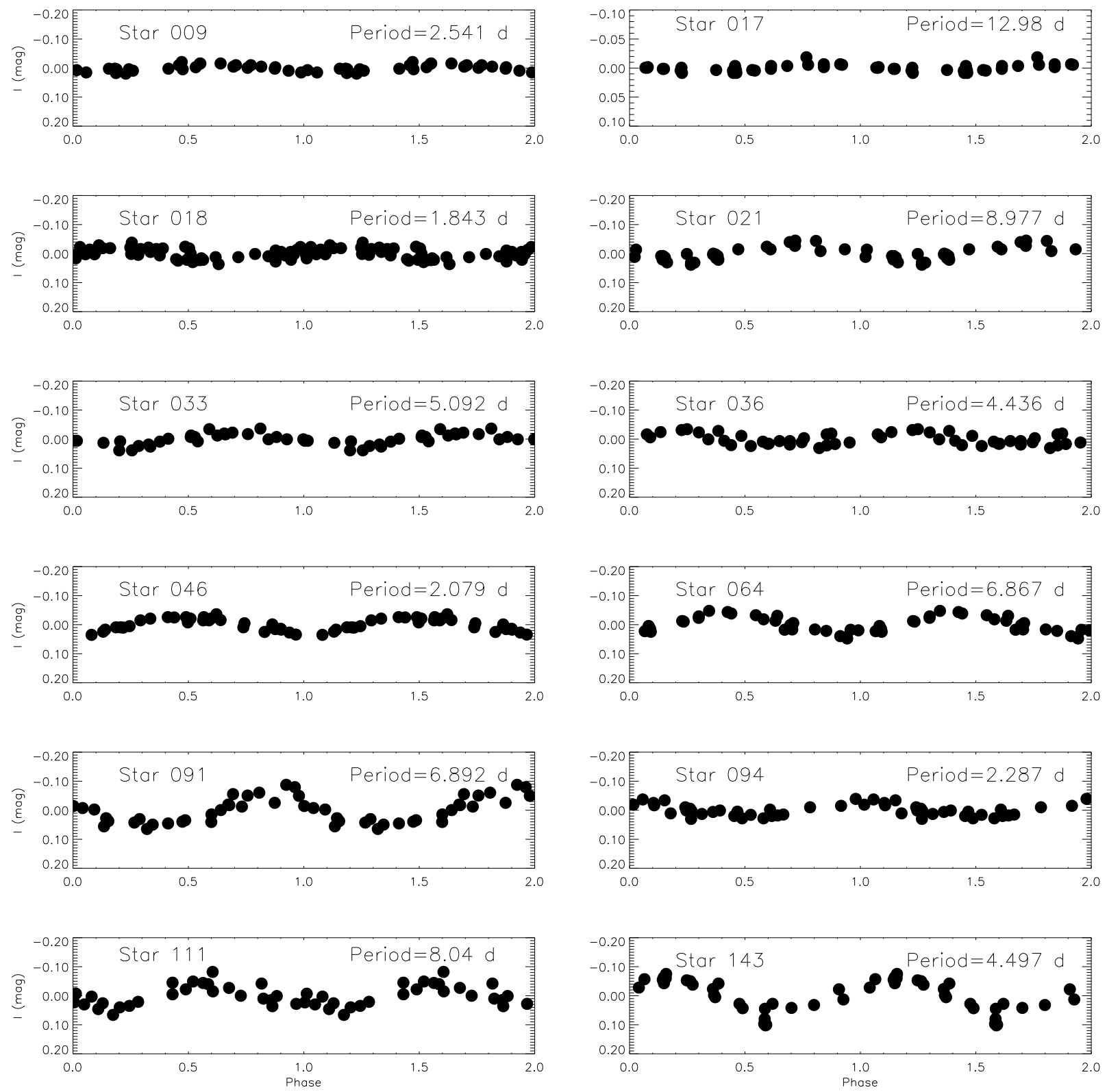

Fig. 2.- Phased light curves for the newly detected periodic stars. All are shown with 2004-2005 data except for star 46, which is shown with 2002-03 data and star 18, which is shown with 1999-2000 data. 

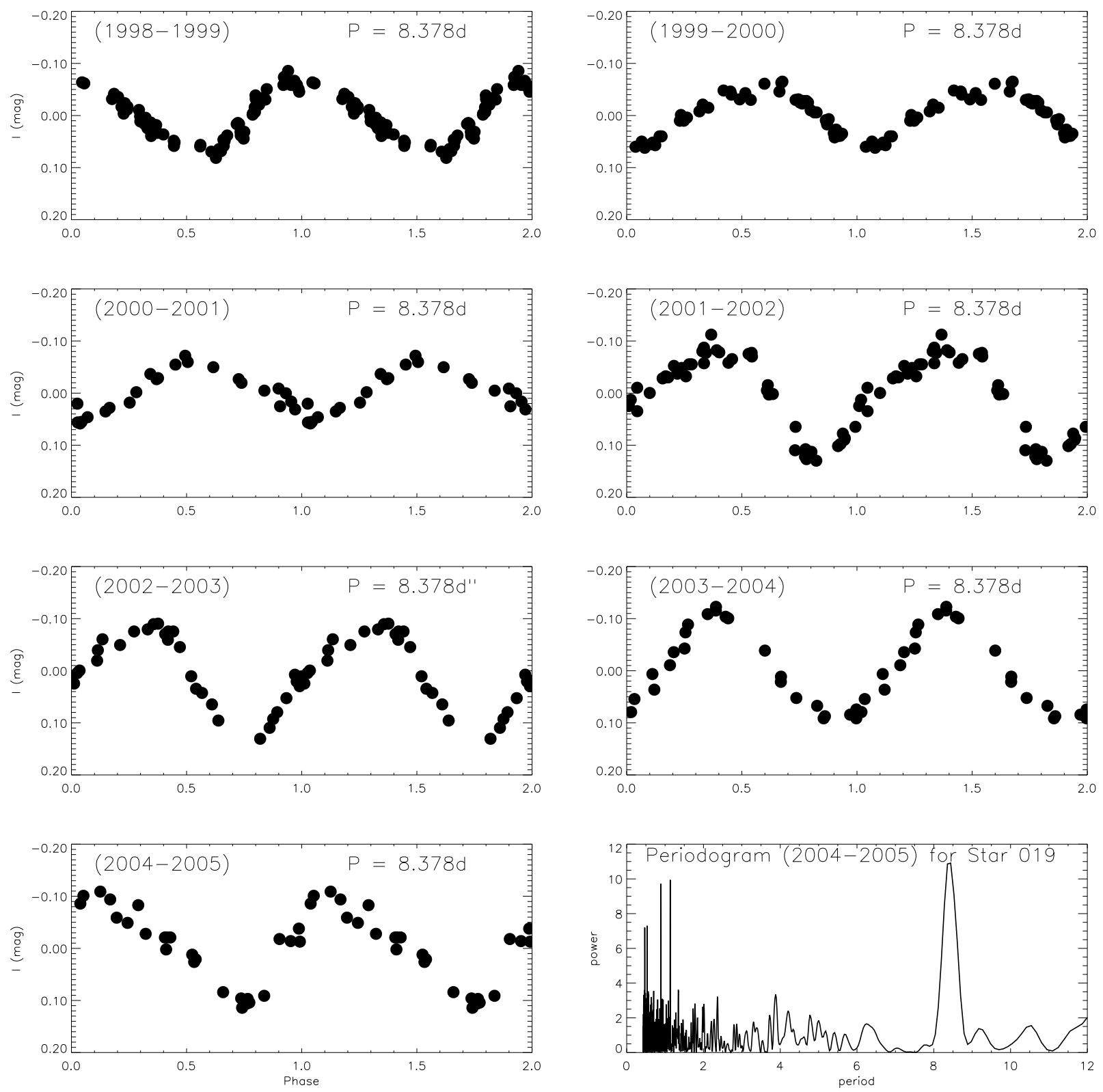

Fig. 3.- Example light curve of a star (number 19) with near constant periodic behavior throughout the 7-year observing period. Changes in light curve shape are still apparent. The final panel shows the periodogram (power vs. period) for 2004-2005. 

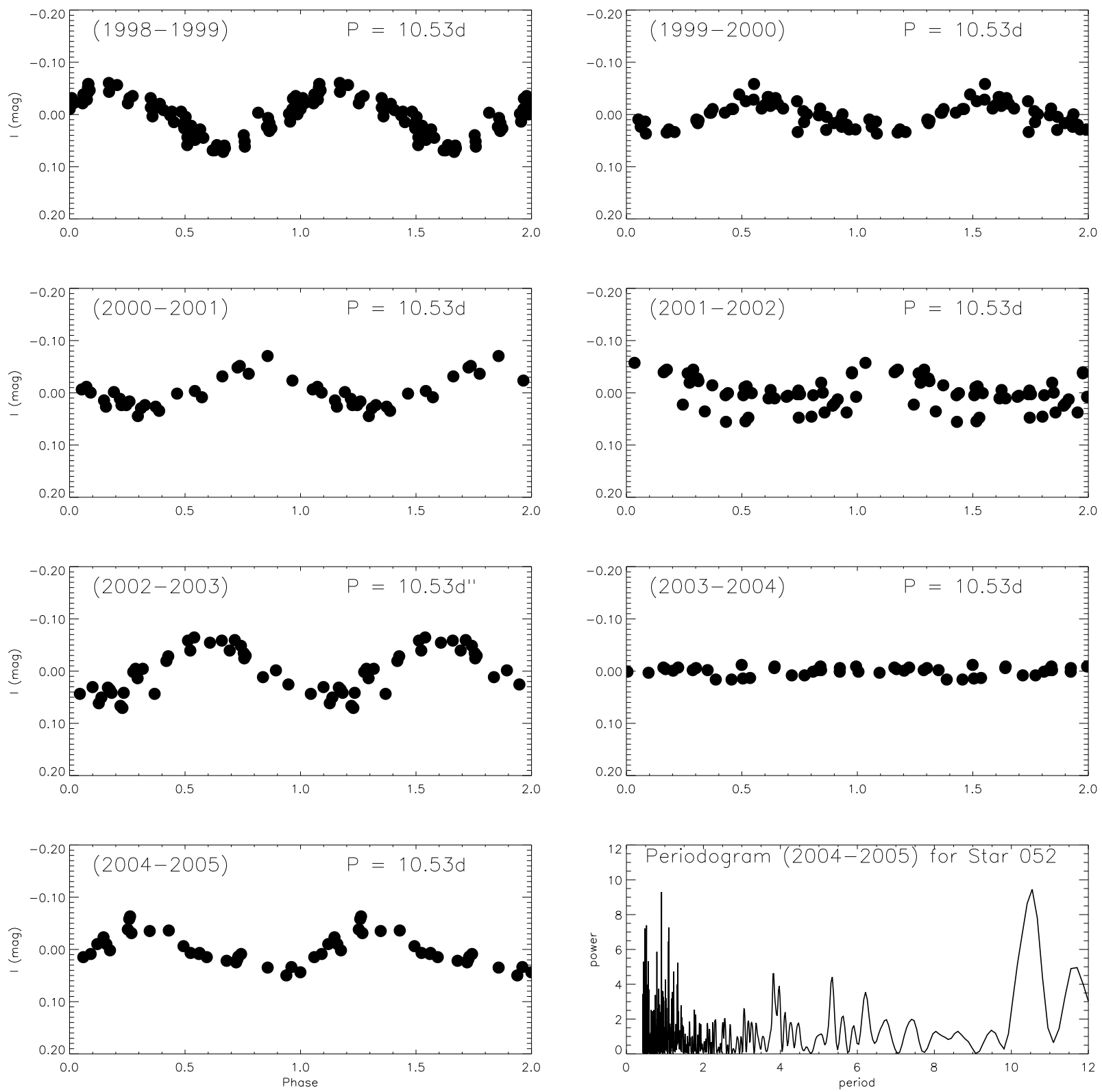

Fig. 4. - Example light curve of a star (number 52) for which the period was not detected in all years. For the nonperiodic years (2001-2 and 2003-4), the data is phased using the average detected period from the other years. The final panel shows the periodogram (power vs. period) for 2004-2005. 

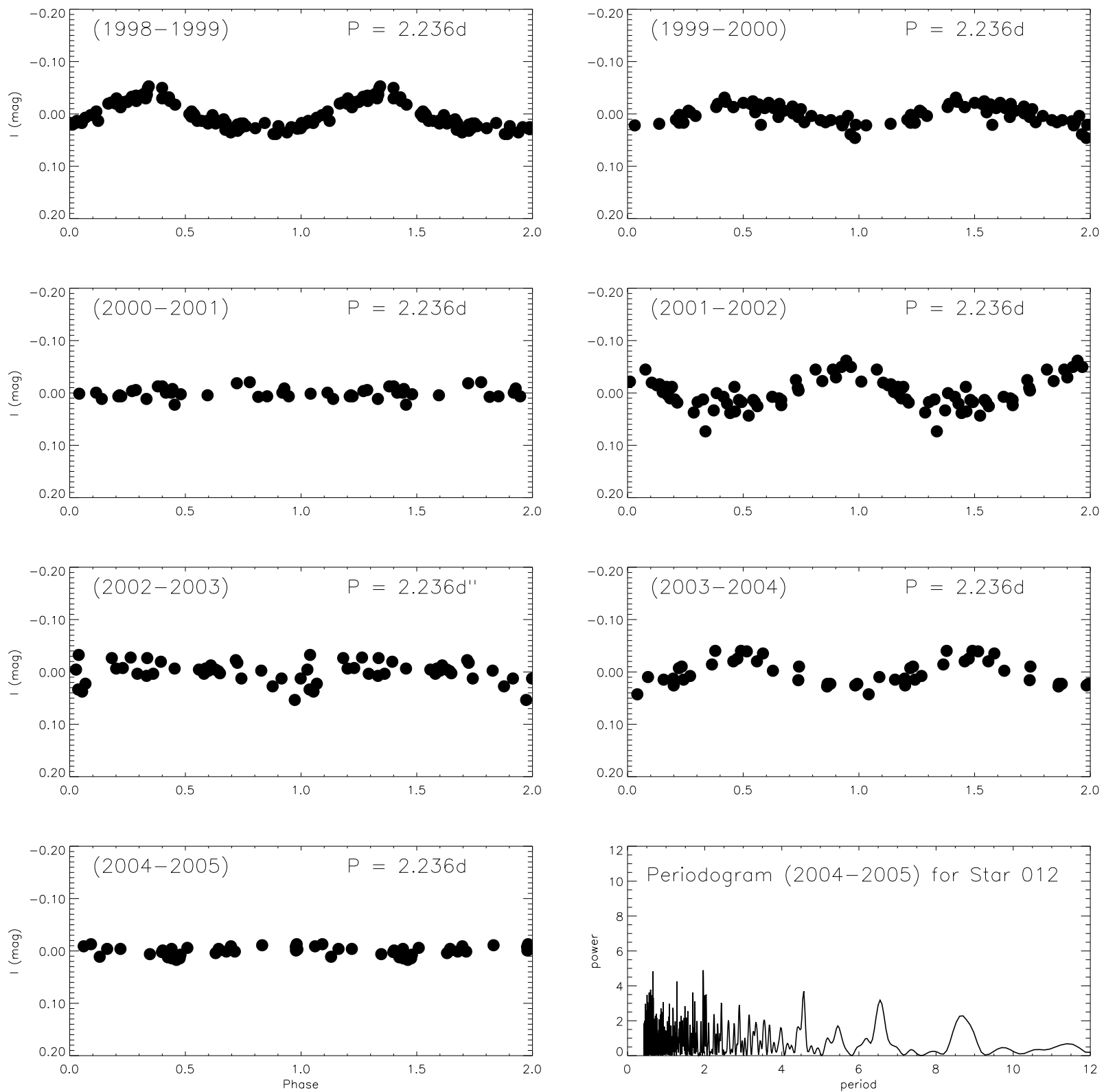

Fig. 5. - Light curves of star 12, over 7 seasons phased with our identified period of $\sim 2.2$ days. Clear periodic structure can be seen in four seasons. The final panel shows the 2004-05 periodogram for the star. Littlefair et al. (2005) report a period of 10.8 days for this star but the data phased with that period show no hint of periodicity in any season. We conclude that their determination is in error and that the actual period of the star is 2.24 days. 

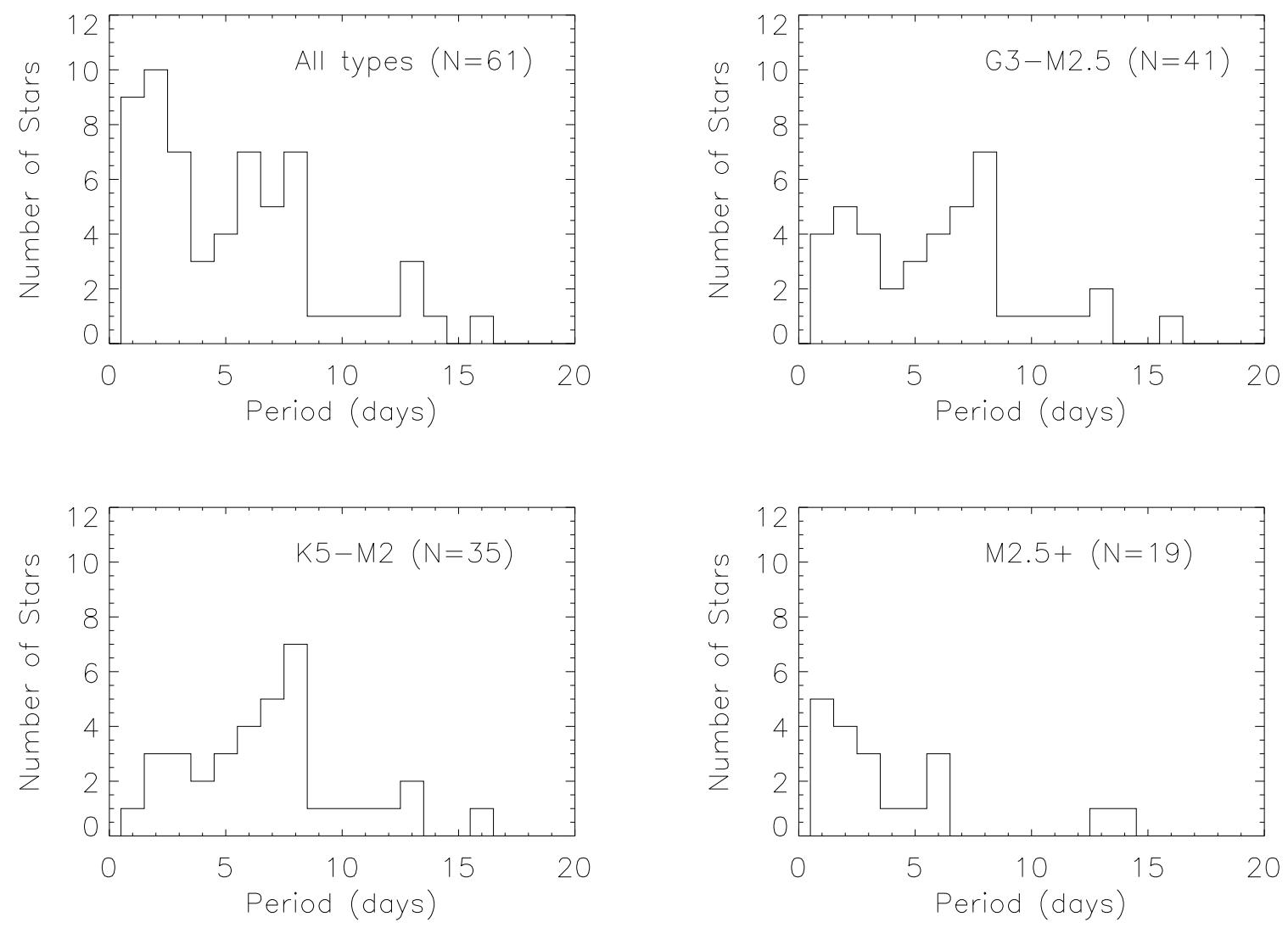

Fig. 6. - Top Left: Distribution of periods for all periodic stars in the sample, including the additional stars found to be periodic by Littlefair et al. (2005). Top Right; Distribution limited to stars of spectral type G3-M2.5. The decrease in fast rotators when later-type stars are eliminated is evident. Bottom Left: Distribution limited to types K5-M2, showing an additional decrease in fast rotators when early-type stars are also eliminated. Bottom Right: Distribution for the 19 stars with spectral types M2.5 and later, many of which are too faint to be detected as periodic by our photometry, and thus have periods measured only by Littlefair et al. (2005). Note that there are no stars in IC 348 with measured periods less than 1 day. 

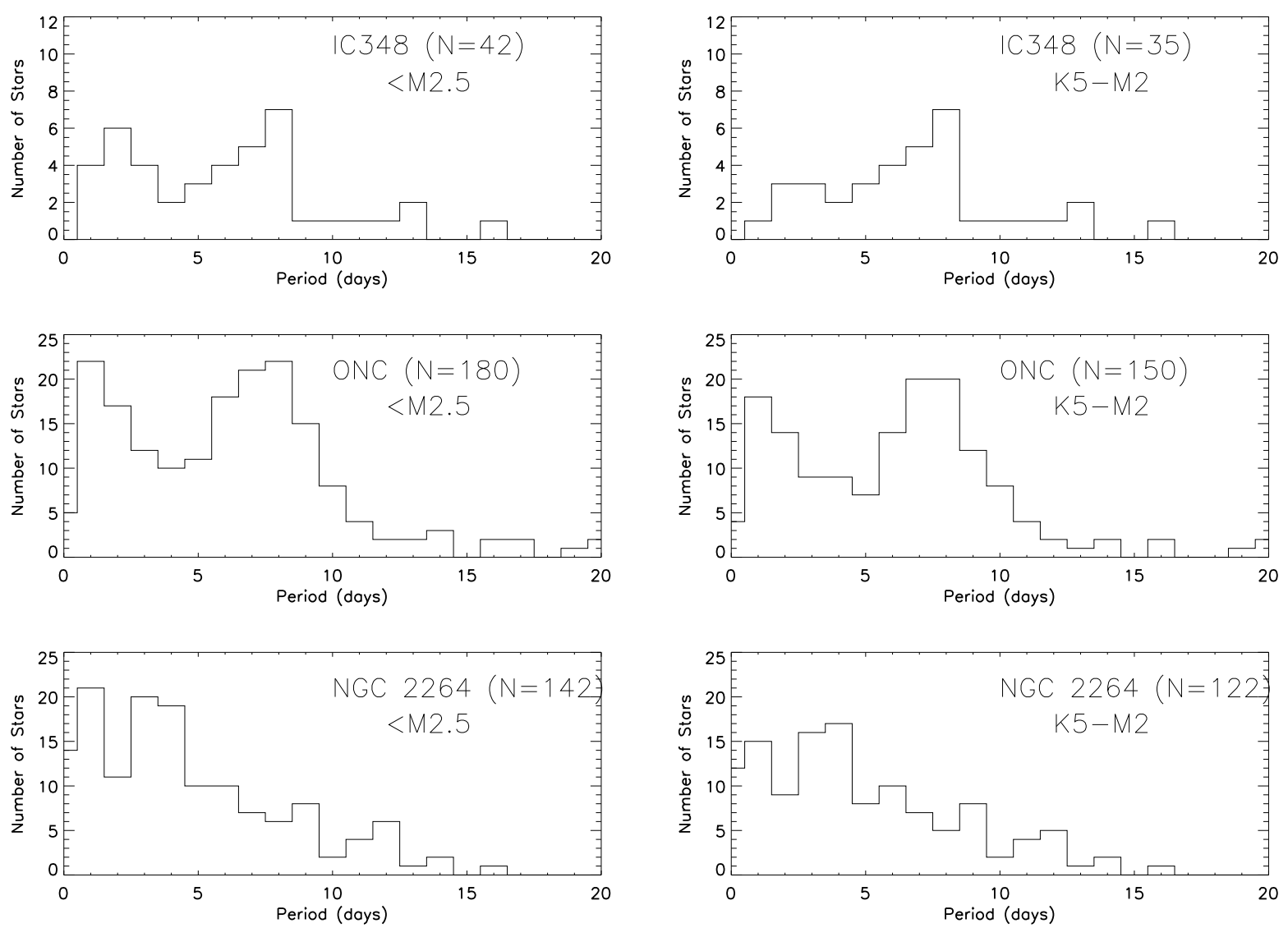

Fig. 7.- Distribution of IC 348 periods (top) compared with those of the Orion Nebula Cluster (ONC - center) and NGC 2264 (bottom) for stars of spectral types earlier than M2.5 (left panels) and between K5 and M2 (right panels). In each case, the IC 348 and ONC distributions show roughly the same distribution and this is confirmed by a KolmogorovSmirnov (K-S) test giving a 72\% (left) and 56\% (right) probability that the two distributions are drawn from the same parent population. On the other hand, differences are seen between the IC 348 and NGC 2264 distributions which are significant at the 2 to 3 sigma level. A two-sided K-S test yields only a $3.3 \%$ (left) or $0.4 \%$ (right) probability that the data for the two different clusters are drawn from the same parent population. Note that there are no stars in IC 348 with measured periods less than 1 day. 

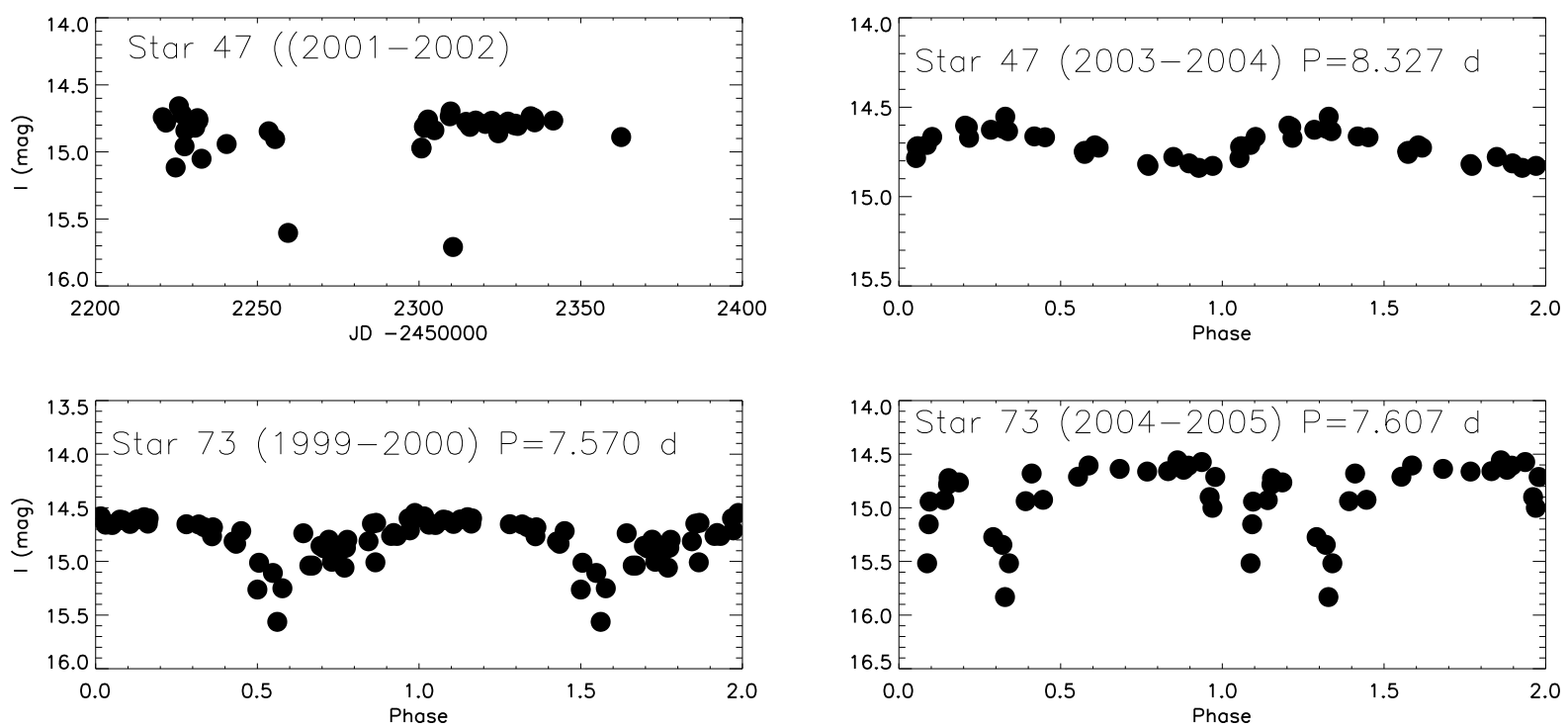

Fig. 8.- Light Curve and Phase plots for the two periodic and irregular stars in our sample. Top: Star 47 shows highly irregular behavior in 2001-2002, characterized by dips in brightness as large as $\sim 1 \mathrm{mag}$ (left), but shows standard moderate-amplitude $(\sim 0.25)$ periodic behavior in 2004-2005 (right.) Bottom: Star 73 shows well-defined but atypical periodic behavior in 1999-2000 (left) but less definitively periodic variations in 2004-2005 (right.) In both seasons the star decreases by about 1.3 magnitudes, a much greater brightness change than any other periodic sample stars. 


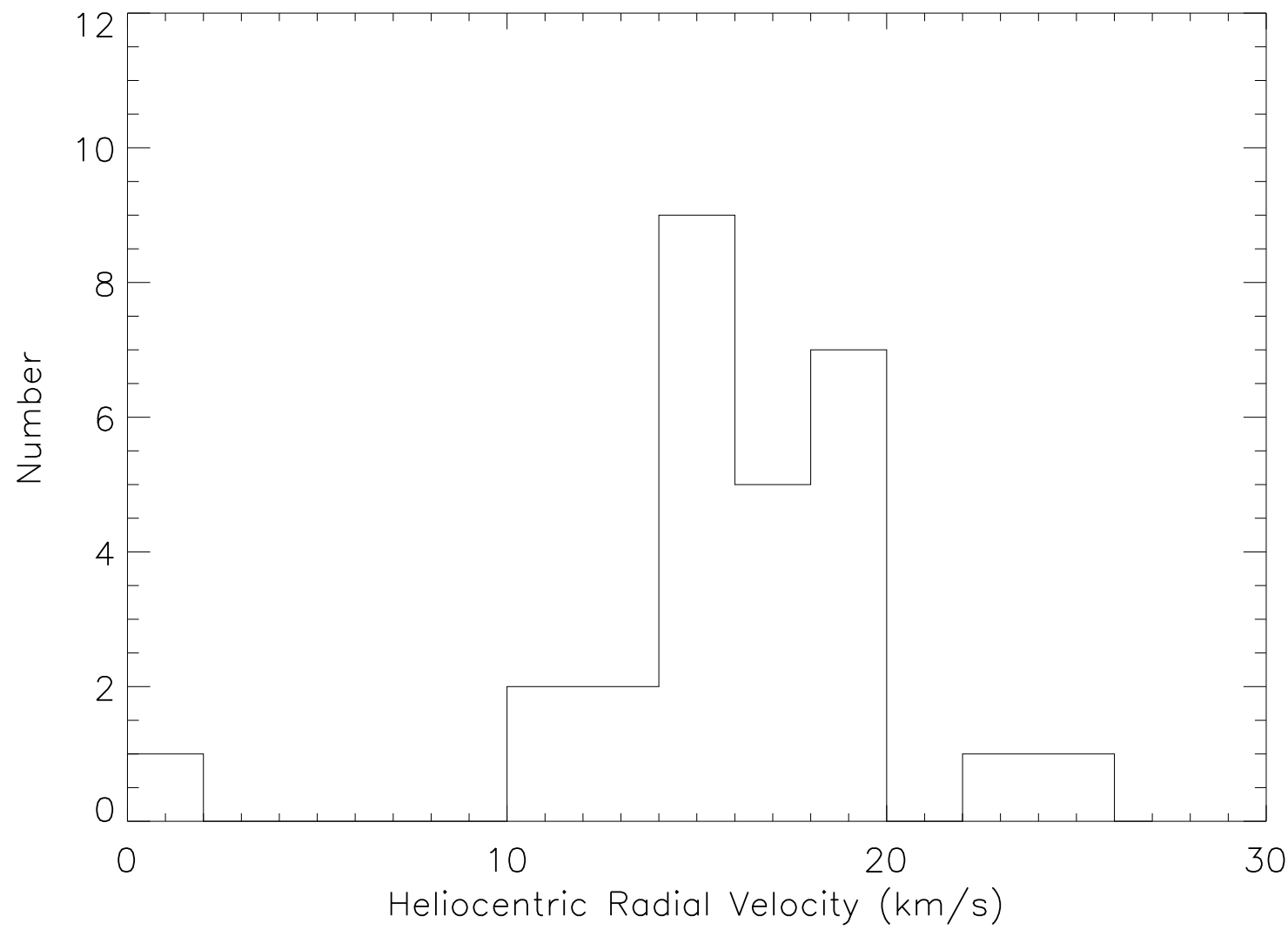

Fig. 9.- Histogram of the measured heliocentric radial velocity values for cluster stars. Two possible SB2's with negative velocities are not shown. The mean and standard deviation calculated omitting those two stars are $16.5 \mathrm{~km} \mathrm{~s}^{-1}$ and $3.1 \mathrm{~km} \mathrm{~s}^{-1}$, respectively. 


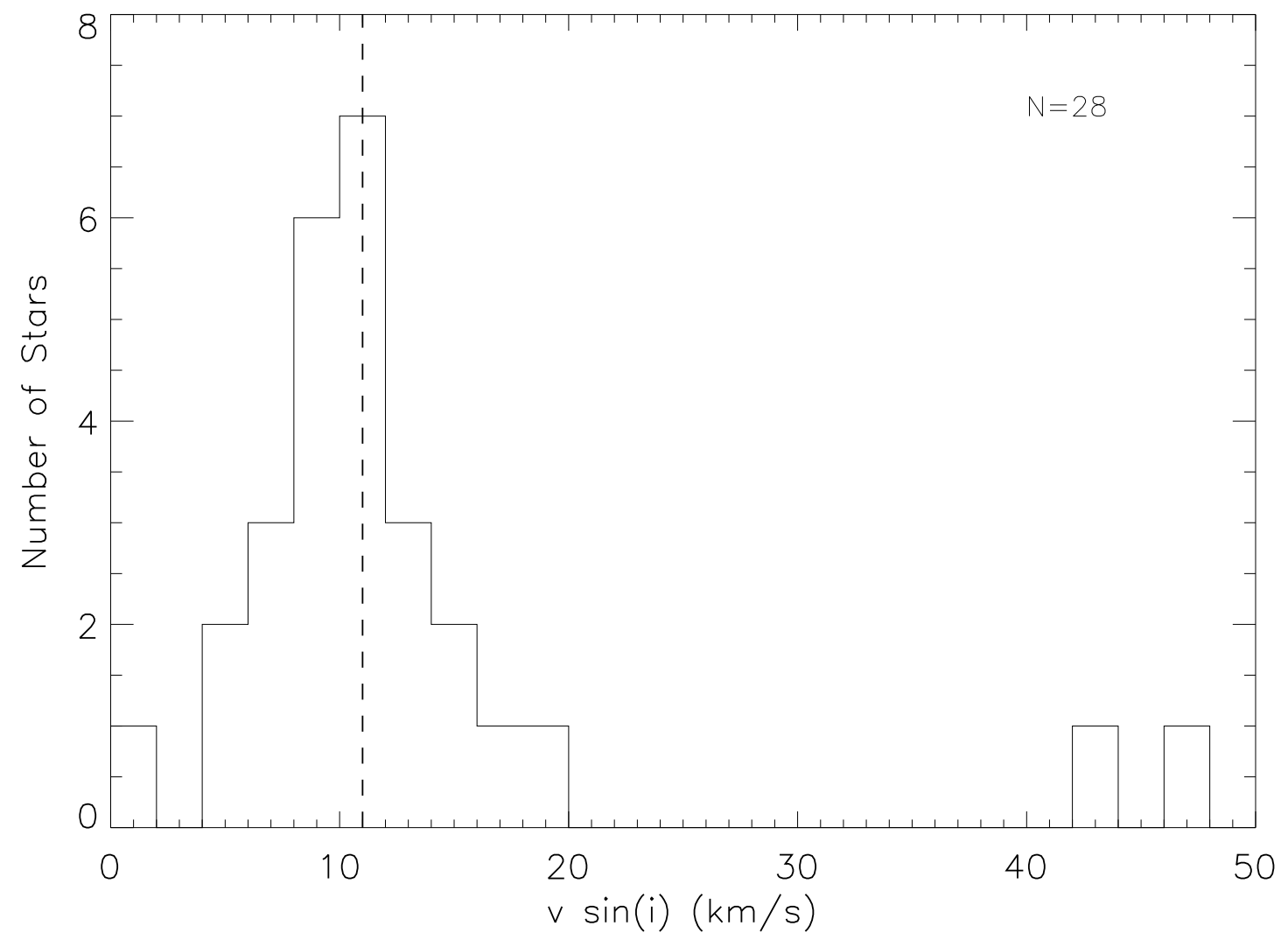

Fig. 10.- Histogram of the distribution of $v \sin i$ for the entire useable sample of 28 stars. The dashed line at $11 \mathrm{~km} / \mathrm{s}$ indicates our estimated measurement limit and values below this have large percentage errors and should be considered only as upper limit measurements. 


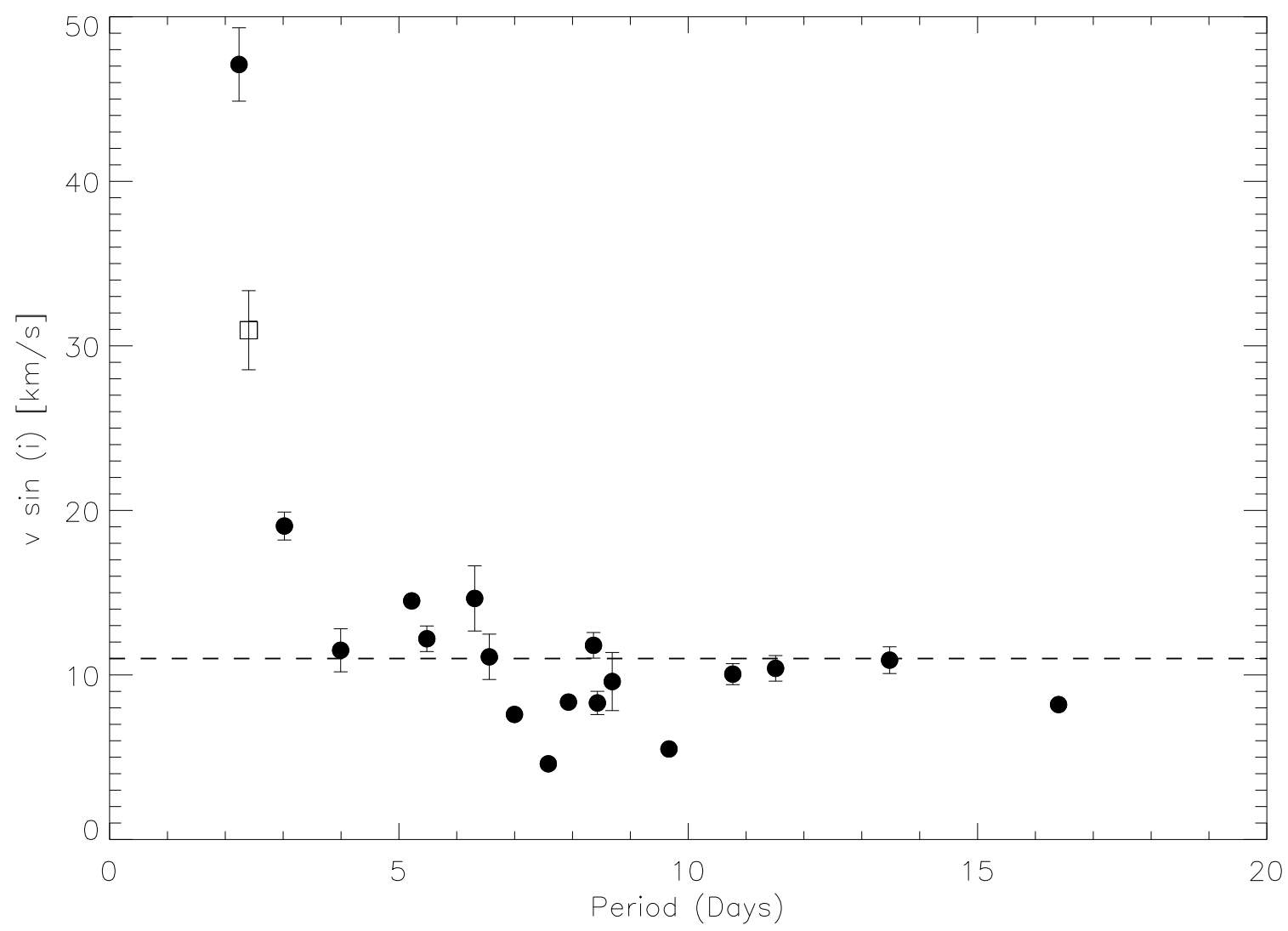

Fig. 11.- Measured $v$ sin $i$ vs. period, showing the expected shape; a possible double-lined spectroscopic binary (based on the line profile) is plotted as a square. The dashed line at 11 $\mathrm{km} / \mathrm{s}$ indicates our estimated measurement limit and values below this have large percentage errors and should be considered only as upper limit measurements. 


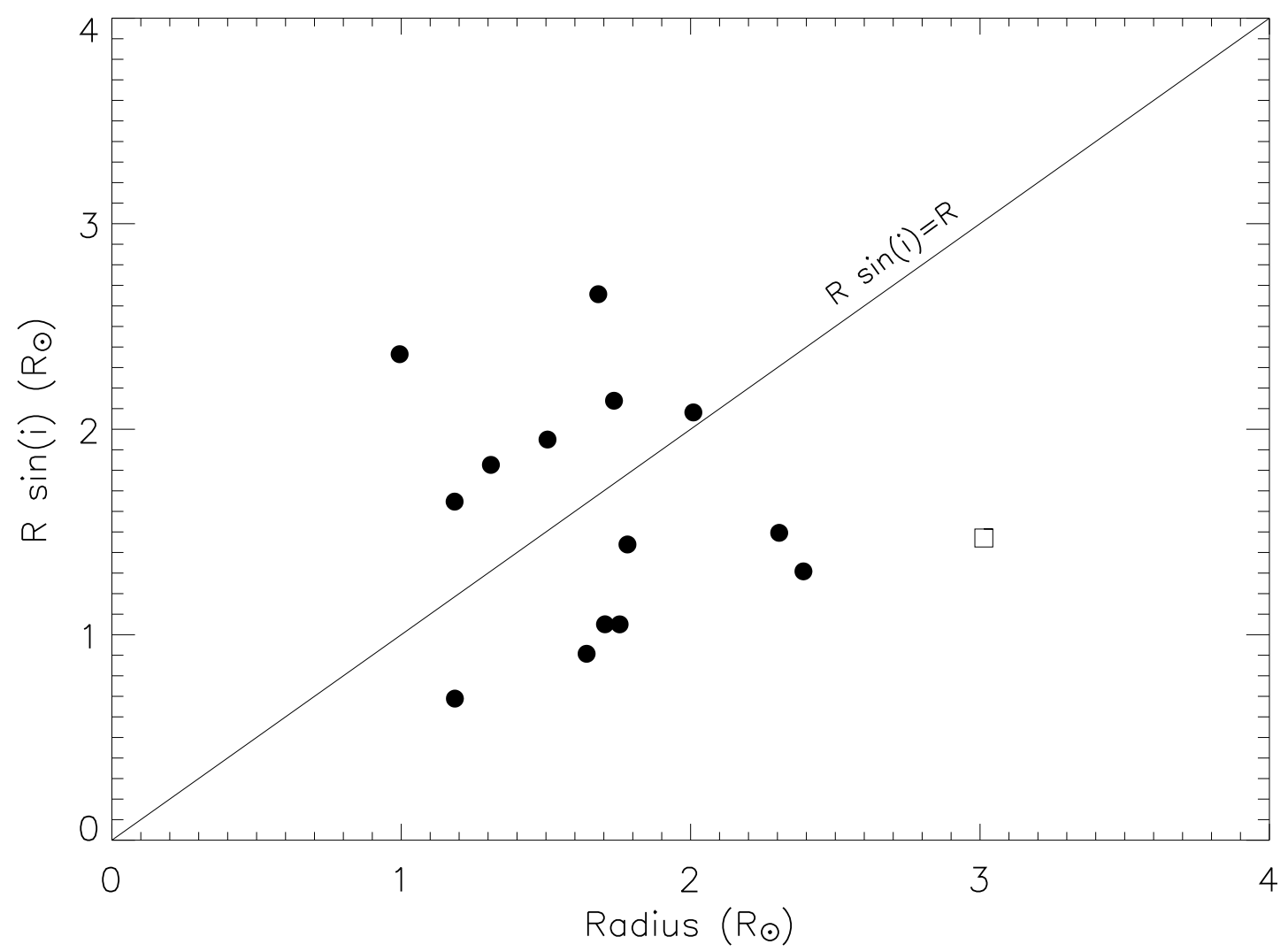

Fig. 12.- Measured $R$ sin $i$ vs. radius, as calculated from luminosity and effective temperature. The solid line is $R \sin i=R$, and the possible spectroscopic binary (SB) is plotted as a square. Note that the SB candidate appears from its $\mathrm{R}$ value to be overluminous, which is another piece of evidence suggesting it might be an SB. 


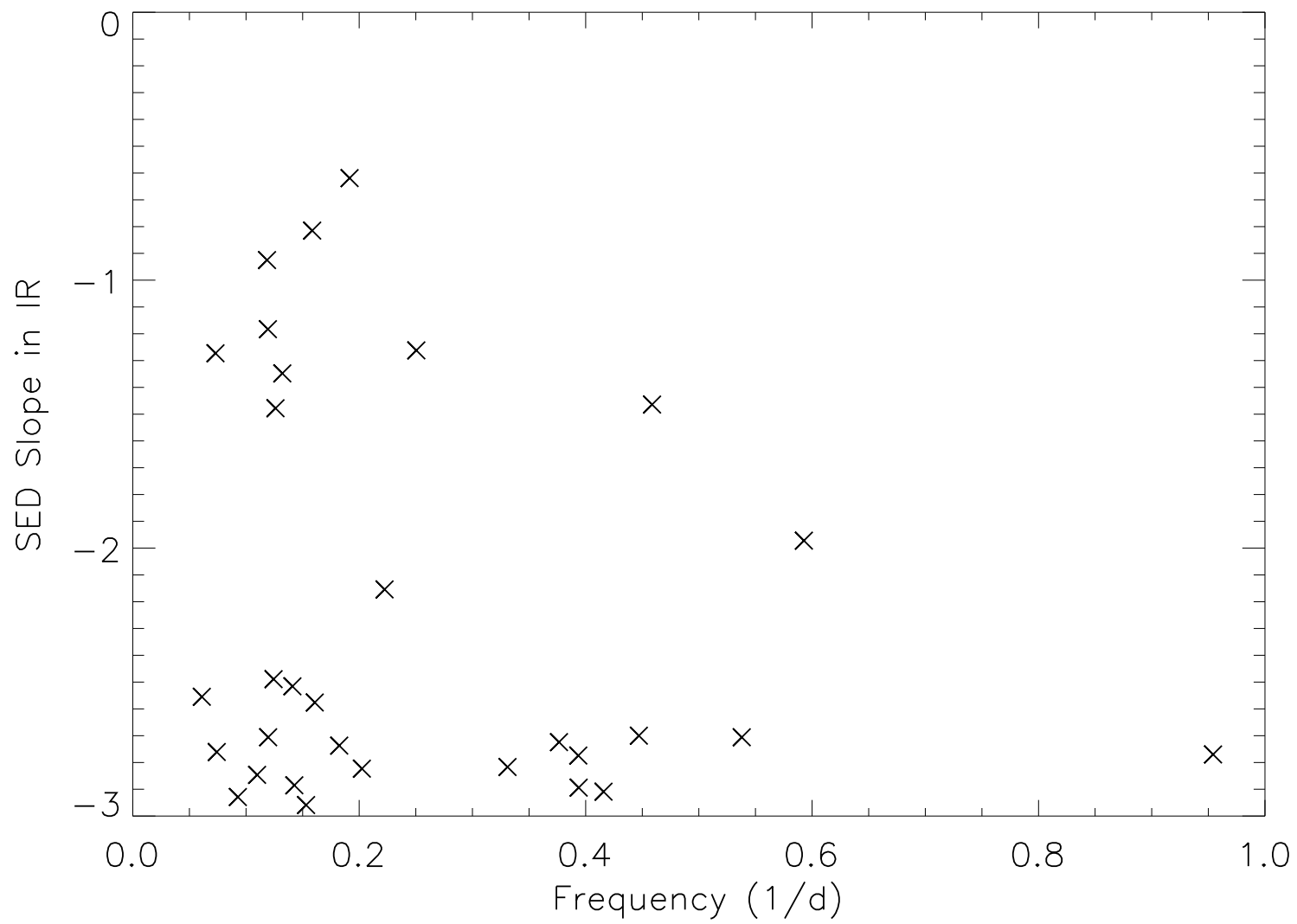

Fig. 13. - The slope of the mid-infrared portion of the spectrum, as defined by Lada et al. (2006) on the basis of their Spitzer data is shown as a function of rotational frequency for $\mathrm{G}$ to M2 stars in IC 348. There is a weak trend for rapid rotators (Period $<\sim 3$ days) to have large negative slopes, indicative of little or no excess emission characteristic of a disk. On the other hand, slow rotators have a higher percentage of stars with shallower slopes, indicative of infrared excesses attributed to circumstellar disks. While the sample size is too small to be statistically significant, the distribution is quite similar to what is found in the much larger sample in the ONC. 


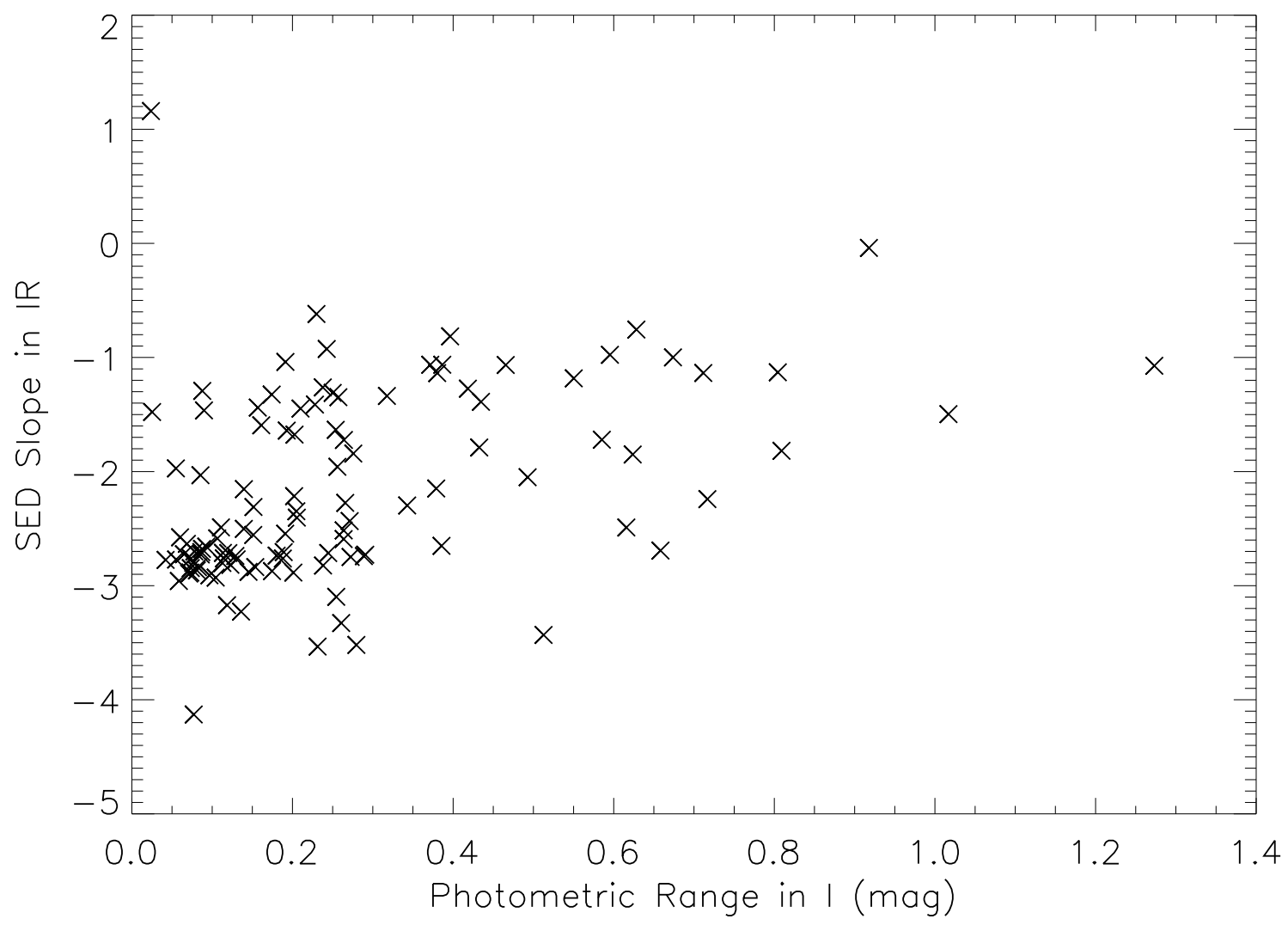

Fig. 14. - The slope of the mid-infrared portion of the spectrum, as defined by Lada et al. (2006) on the basis of their Spitzer data is shown as a function of photometric range for $\mathrm{G}, \mathrm{K}$ and $\mathrm{M}$ stars in IC 348. There is a clear trend for large amplitude variables to have less negative slopes, indicative of excess emission characteristic of a disk. Virtually all stars with photometric ranges exceeding $0.4 \mathrm{mag}$ in I have evidence for disks. Large amplitude photometric variability is an excellent predictor of IR excess in this sample. On the other hand, IR excess emission by itself does not mean a star will necessarily be a large amplitude photometric variable. Absence of IR excess emission does mean that a star in this cluster is very unlikely to be photometrically active at a level exceeding $0.3 \mathrm{mag}$ in I. 